\title{
Polaronic effects in one- and two-band quantum systems
}

\author{
Tao Yin,,${ }^{1, *}$ Daniel Cocks,${ }^{2}$ and Walter Hofstetter ${ }^{1}$ \\ ${ }^{1}$ Institut für Theoretische Physik, Goethe-Universität, 60438 Frankfurt/Main, Germany \\ ${ }^{2}$ College of Science, Technology 86 Engineering, James Cook University, Townsville 4810, Australia
}

(Dated: June 18, 2021)

\begin{abstract}
In this work we study the formation and dynamics of polarons in a system with a few impurities in a lattice immersed in a Bose-Einstein condensate (BEC). This system has been experimentally realized using ultracold atoms and optical lattices. Here, we consider a two-band model for the impurity atoms, along with a Bogoliubov approximation for the BEC, with phonons coupled to impurities via both intraband and interband transitions. We decouple this Fröhlich-type term by an extended two-band Lang-Firsov polaron transformation using a variational method. The new effective Hamiltonian with two (polaron) bands differs from the original Hamiltonian by modified coherent transport, polaron energy shifts, and induced long-range interaction. A Lindblad masterequation approach is used to take into account residual incoherent coupling between polaron and bath. This polaronic treatment yields a renormalized inter-band relaxation rate compared to Fermi's golden rule. For a strongly coupled two-band Fröhlich Hamiltonian, the polaron is tightly dressed in each band and can not tunnel between them, leading to an inter-band self-trapping effect.
\end{abstract}

PACS numbers: 67.85.-d, 71.38.-k, 03.65.Yz, 63.20.K-

\section{INTRODUCTION}

The field of ultracold atom physics has explored a wide variety of phenomena since its relatively recent accessibility, with a major feature being the tunability of experiments across wide parameter regimes [1-4], to easily access and probe phase transitions [5,6], as well as excitation spectra and dynamics of systems analog to condensed matter [7-10]. Even features such as artificial gauge fields can be implemented for neutral atomic particles, allowing for the investigation of topological phases $[11,12]$.

Within this ultracold toolbox, one ingredient is becoming of increasing interest in recent years which is of vital importance to real solid-state systems: phonons and atom-phonon coupling [13-16]. Such a coupling provides many interesting possibilities [17-19]. For one, it can lead to effective Hamiltonians, such as extended Hubbard models or the Holstein model [20-24], as well as dissipative two-level system [25-28]. Polaronic effects from electron-phonon interactions have also long been suggested to be the proponent behind high- $T_{c}$ superconductivity in one- and two-band solid-state systems [2935]. In ultracold quantum gases, evidence of polarons has been found in systems with trapped ions [36] or systems with a single ion immersed in a degenerate quantum gas [37-43]. On the other hand, the atomic polaron has also been studied both experimentally and theoretically in systems of imbalanced Bose-Fermi mixtures [44-49] and Fermi-Fermi mixtures [50-57]. In the particular case of a system with impurities immersed in a bosonic bath, these impurities couple to bosonic excitations. For suitable parameters, polaronic phenomena arise generically

*Electronic address: taoyin@itp.uni-frankfurt.de in such systems [58-81]. There are also other proposals for realizing atom-phonon couplings and polaronic effects, including crystals of dipolar molecules [82-84], nanoparticles $[85,86]$ and hybrid atom-ion coupled systems [87].

None of these works consider polaronic phenomena of multi-band systems with ultracold quantum gases. In such systems, the inter-band dynamics of polarons, as well as intra-band dynamics, lead to new effects which have no analogue in single band systems. Motivated by recent experiments [88-91], we consider a system of a few impurities in an optical lattice, populating the lowest two Bloch bands, immersed in a Bose-Einstein condensate. These impurities are coupled to Bogoliubov phonons (of the BEC) via both intra- and inter-band transitions. In order to decouple this Fröhlich-like term, we derive a generalized two-band Lang-Firsov polaron transformation. The transformed effective Hamiltonian still contains two bands, where the impurity is now dressed by phonons as a quasi-particle (polaron). We use a variational approach to connect between the weak and strong coupling limits and calculate the dressing parameters. Polaronic effects modify both intra-band coherent transport and polaron energy shifts, and also induce a long-range interaction between different polarons.

We then focus on inter-band relaxation effects and specify our system as a single impurity trapped in a quasi1D system. We study the residual incoherent coupling between polaron and bath by using a Lindblad master equation. The impurity inter-band relaxation process under this polaronic treatment is beyond a Fermi's Golden Rule description. These polaronic renormalization effects of the inter-band relaxation rate should be accessible in current experiments. On the other hand, for large impurity-phonon coupling, the polaron is tightly dressed in each band and cannot hop between different bands. In this limit, an inter-band self-trapping effect is expected. 
This work is organized as follow: in section II, we introduce the effective two-band Hamiltonian of a realistic experiment setup, with a few impurities in a lattice, immersed in a Bose-Einstein condensate (BEC) of a different atomic species. In section III, we describe in detail the generalized Lang-Firsov polaron transformation for the two-band system. The transformed Hamiltonian with two (polaron) bands can be separated into a coherent part and an incoherent part. The coherent part, arising from the thermal average over the phonon bath, is discussed in section IV. The inter-band relaxation and decoherence effects, which are all included by the incoherent part of this Hamiltonian, are discussed in section V. We derive the Lindblad equation and correlation functions for the residual incoherent impurity-phonon coupling. This polaronic impurity dynamics is closely related to recent experiments. The polaronic inter-band relaxation rate is compared to Fermi's Golden Rule. We give concluding remarks and an outlook in section VI.

\section{EFFECTIVE HAMILTONIAN}

Here we consider a few neutral impurities with mass $m_{I}$ interacting with a Bose-Einstein condensate of another neutral species. The impurities are trapped by a 3D optical lattice and their Hamiltonian is denoted by $H_{I}$. The homogeneous BEC system $H_{B}$ is formed by another atomic species with mass $m_{B}$ and a weak repulsive interaction $g_{B}$. The impurity-BEC interaction $H_{\text {int }}$ is caused by $s$-wave interactions between the different species, which can be tuned by standard Feshbach resonance techniques. The total Hamiltonian is hence

$$
H=H_{I}+H_{B}+H_{\text {int }} .
$$

We describe these different terms in detail in the following parts of this section.

\section{A. Impurities in optical lattice $-H_{I}$}

We consider a quasi-1D system with impurities trapped in an anisotropic 3D optical lattice:

$$
V_{I}(\mathbf{r})=V_{I}^{x} \sin ^{2}\left(\frac{\pi}{d} x\right)+V_{I}^{y} \sin ^{2}\left(\frac{\pi}{d} y\right)+V_{I}^{z} \sin ^{2}\left(\frac{\pi}{d} z\right),
$$

with lattice constant $d=\lambda / 2$ and laser wavelength $\lambda$. We use the lattice constant $d$ as the unit of length throughout this paper. The single impurity recoil energy is $E_{R} \equiv$ $\pi^{2} \hbar^{2} /\left(2 m_{I}\right)$. The trapping strength in the transverse $(y, z-)$ direction is assumed to be much stronger than in longitudinal $(x-)$ direction with $V_{I}^{x} \ll V_{I}^{\perp} \equiv V_{I}^{y}=$ $V_{I}^{z}$. The impurities are therefore tightly trapped in the transverse direction, and remain in the ground state of the associated harmonic oscillator potential:

$$
\phi^{0}(y)=1 /\left(\pi \sigma_{\perp}^{2}\right)^{\frac{1}{4}} e^{-y^{2} /\left(2 \sigma_{\perp}^{2}\right)},
$$

with transverse characteristic length $\sigma_{\perp} \equiv \sqrt{\hbar /\left(m_{I} \omega_{\perp}\right)}$ and frequency $\hbar \omega_{\perp} \equiv 2\left(V_{I}^{\perp} E_{R}\right)^{1 / 2}$. In the longitudinal direction, on the other hand, the impurities populate both the lowest and the first excited band. In principle, exact Bloch wave functions need to be calculated numerically, which can then also be represented as linear combinations of Wannier states $\varphi_{x}^{0(1)}(x)$. In a deep lattice, the Wannier functions can be approximated by the lowest two eigenstates of a harmonic oscillator

$$
\begin{aligned}
& \varphi_{x}^{0}(x) \approx 1 /\left(\pi \sigma_{x}^{2}\right)^{\frac{1}{4}} e^{-x^{2} /\left(2 \sigma_{x}^{2}\right)} \\
& \varphi_{x}^{1}(x) \approx 1 /\left(\pi \sigma_{x}^{2}\right)^{\frac{1}{4}}\left(\sqrt{2} x / \sigma_{x}\right) e^{-x^{2} /\left(2 \sigma_{x}^{2}\right)},
\end{aligned}
$$

with longitudinal characteristic length $\sigma_{x} \equiv \sqrt{\hbar /\left(m_{I} \omega_{x}\right)}$ and oscillation frequency $\hbar \omega_{x} \equiv 2\left(V_{I}^{x} E_{R}\right)^{1 / 2}$.

In this highly anisotropic system, impurities can hop to their nearest-neighbor sites only along the longitudinal direction. A particle localized on lattice site $j$ will be described by the wave function

$$
W_{j}^{0(1)}(\mathbf{r}) \equiv \varphi_{x}^{0(1)}\left(x-x_{j}\right) \phi^{0}(y) \phi^{0}(z) .
$$

Due to the low density of impurities, their dynamics can be modeled by a non-interacting two-band Hubbard Hamiltonian:

$$
\hat{H}_{I}=-\sum_{\langle i, j\rangle} \sum_{\alpha} J^{\alpha} \hat{a}_{i}^{\alpha \dagger} \hat{a}_{j}^{\alpha}+\sum_{i} \sum_{\alpha} \varepsilon^{\alpha} \hat{n}_{i}^{\alpha}
$$

where $J^{\alpha}$ and $\varepsilon^{\alpha}$ are the hopping parameters and on-site energy for each band with index $\alpha=0,1$. In a deep lattice, the band gap $\varepsilon^{\Delta} \equiv \varepsilon^{1}-\varepsilon^{0}$ can be approximated as the longitudinal oscillator frequency $\hbar \omega_{x}$. In this work we only consider inter-band dynamics between the lowest two Bloch bands and ignore higher band effects.

\section{B. Bosonic bath}

The impurities are immersed in a homogeneous BEC with weakly repulsive boson-boson interaction $g_{B}$ between the atoms. In a dilute system, this weak interaction can be described by boson-boson scattering length $a_{B}$ as $g_{B}=4 \pi \hbar^{2} a_{B} / m_{B}$. For vanishing inter-species interaction $g_{I B}$ between impurity and bath, the BEC can be described by standard Bogoliubov theory and treated as a phonon bath (see Fig. 1). Once $g_{I B}$ is introduced, the BEC becomes deformed due to the presence of impurities. This interaction is closely related to the impurity-boson scattering length and other system parameters such as the impurity-boson mass ratio and the impurity confinement strength. The relation can be determined by making use of scattering theory in the low-energy limit, such as the Lippmann-Schwinger equation or effective field theory [92]. For an unconfined impurity, the inter-species interaction $g_{I B}$ can be derived as $g_{I B} \equiv 2 \pi \hbar^{2} a_{I B} / \mu$ with the reduced mass 


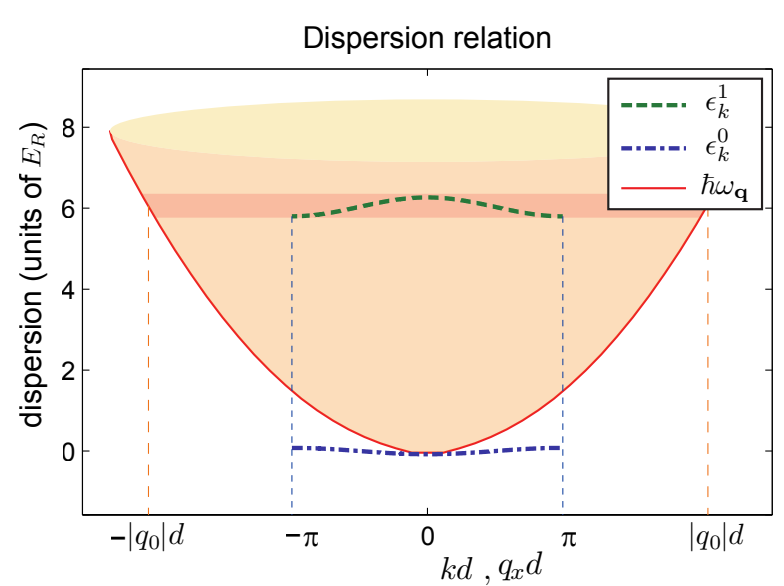

Figure 1: Dispersion relation for single impurity and phonon in longitudinal direction (in the unit of $E_{R}$ ). The impurity moves in the longitudinal direction of the system with two Bloch bands $\epsilon_{k}^{0}$ (blue line) and $\epsilon_{k}^{1}$ (green line). The dashed blue lines at $k= \pm \pi$ indicate the first Brillouin zone in longitudinal direction. The phonon dispersion $\hbar \omega_{\mathbf{q}}$ (red line) is described by Bogoliubov theory where $\mathbf{q} \equiv\left(q_{x}, q_{y}, q_{z}\right)$ is the phonon momentum. The dashed red lines at $q_{x}= \pm\left|q_{0}\right|$ indicate that the phonon energy matches with the impurity band gap $\hbar \omega_{\left|q_{0}\right|} \approx \varepsilon^{\Delta}$ with $\varepsilon^{\Delta} \equiv \varepsilon^{1}-\varepsilon^{0}$.

$\mu \equiv m_{I} m_{B} /\left(m_{I}+m_{B}\right)$ and 3D impurity-boson scattering length $a_{I B}$. On the other hand, for the confined impurity, $g_{I B}$ needs to be treated carefully due to lattice effects such as confinement induced resonances [93, 94]. In the specific system we considered here, the impurity is confined in one-dimensional tube (quasi1D) by an anisotropic optical lattice while the bosonic atoms are free in three-dimensional space (3D). When the transverse characteristic length $\sigma_{\perp}$ is much smaller than any other length scales, the resulting system is mixeddimensional. At low energies, the inter-species interaction is solely characterized by a single parameter, the effective scattering length $a_{I B}^{\mathrm{eff}}$, whose value can be obtained numerically [95]. This fact also allow us to arbitrarily tune the value of $a_{I B}^{\text {eff }}$ by tuning the transverse confinement strength, independently of tuning the Feshbach resonance position.

Here we use the approach in $[60,61]$, where the deformation is treated as a perturbation around the BEC ground state. The bosonic field operator is expanded as $\hat{\psi}(\mathbf{r})=\psi_{0}(\mathbf{r})+\hat{\vartheta}(\mathbf{r})$, where $\psi_{0}(\mathbf{r})$ is the order parameter in the absence of inter species interaction and $\hat{\vartheta}(\mathbf{r})=\vartheta(\mathbf{r})+\hat{\zeta}(\mathbf{r})$ represents the perturbation itself, which consists of a correction to the order parameter, $\vartheta(\mathbf{r})$, and Bogoliubov excitation operators $\hat{\zeta}(\mathbf{r})$. The modified BEC ground state is described as $\psi_{0}(\mathbf{r})+\vartheta(\mathbf{r})$, which is the Gross-Pitaevskii solution including the presence of impurities. This modification $\vartheta(\mathbf{r})$ shifts the equilibrium positions in order to minimize the total energy of the system. The small excitations $\hat{\zeta}(\mathbf{r})$, around the static GP ground state $\psi_{0}(\mathbf{r})+\vartheta(\mathbf{r})$ of the condensate, can be described in the terms of Bogoliubov modes

$$
\hat{\zeta}(\mathbf{r})=\sum_{\mathbf{q}}\left[u_{\mathbf{q}} e^{i \mathbf{q} \cdot \mathbf{r}} \hat{\beta}_{\mathbf{q}}-v_{\mathbf{q}}^{*} e^{-i \mathbf{q} \cdot \mathbf{r}} \hat{\beta}_{\mathbf{q}}^{\dagger}\right]
$$

with bosonic operators $\hat{\beta}_{\mathbf{q}}^{\dagger}\left(\hat{\beta}_{\mathbf{q}}\right)$ creating (annihilating) a Bogoliubov quasi-particle with momenta $\mathbf{q}$. The coefficients $u_{\mathbf{q}}(\mathbf{r})$ and $v_{\mathbf{q}}(\mathbf{r})$ can be determined by Bogoliubovde Gennes equations:

$$
\begin{aligned}
& u_{\mathbf{q}}=\sqrt{1 /(2 \Omega)\left[\left(\epsilon_{\mathbf{q}}+g_{B} n_{0}\right) /\left(\hbar \omega_{\mathbf{q}}\right)+1\right]} \\
& v_{\mathbf{q}}=\sqrt{1 /(2 \Omega)\left[\left(\epsilon_{\mathbf{q}}+g_{B} n_{0}\right) /\left(\hbar \omega_{\mathbf{q}}\right)-1\right]}
\end{aligned}
$$

with the system quantization volume $\Omega$ and the Bogoliubov phonon dispersion

$$
\begin{aligned}
\hbar \omega_{\mathbf{q}} & \equiv \sqrt{\epsilon_{\mathbf{q}}\left(\epsilon_{\mathbf{q}}+2 g_{B} n_{0}\right)} ; \\
\epsilon_{\mathbf{q}} & \equiv \frac{\hbar^{2}|\mathbf{q}|^{2}}{2 m_{B}} .
\end{aligned}
$$

where $n_{0}=\left|\psi_{0}\right|^{2}$ is the condensate density.

As pointed out in [61], the coefficients $u_{\mathbf{q}}(\mathbf{r}), v_{\mathbf{q}}(\mathbf{r})$ and Bogoliubov phonon spectrum $\omega_{\mathbf{q}}$ are not changed by the presence of the impurity, although the equilibrium positions of the modes are modified by $\vartheta(\mathbf{r})$. In [61], they express $\hat{\vartheta}(\mathbf{r})=\vartheta(\mathbf{r})+\hat{\zeta}(\mathbf{r})$ in terms of Bogoliubov modes around the state $\psi_{0}(\mathbf{r})$ as

$$
\hat{\vartheta}(\mathbf{r})=\sum_{\mathbf{q}}\left[u_{\mathbf{q}} e^{i \mathbf{q} \cdot \mathbf{r}} \hat{b}_{\mathbf{q}}-v_{\mathbf{q}}^{*} e^{-i \mathbf{q} \cdot \mathbf{r}} \hat{b}_{\mathbf{q}}^{\dagger}\right]
$$

In contrast to Eq. (6), the bosonic operators $\hat{b}_{\mathbf{q}}^{\dagger}\left(\hat{b}_{\mathbf{q}}\right)$ create (annihilate) a quasi-particle around the ground state $\psi_{0}(\mathbf{r})$ in absence of impurity rather than around state $\psi_{0}(\mathbf{r})+\vartheta(\mathbf{r})$. The Hamiltonian (up to constant terms) for this effective phonon bath can be simply expressed as

$$
\hat{H}_{B}=\sum_{\mathbf{q}} \hbar \omega_{\mathbf{q}} \hat{b}_{\mathbf{q}}^{\dagger} \hat{b}_{\mathbf{q}}
$$

if the impurity-BEC coupling $g_{I B}$ satisfies condition $|\langle\vartheta(\mathbf{r})\rangle| \ll \psi_{0}(\mathbf{r})$, which implies the dimensionless relation:

$$
\left(\left|g_{I B}\right| / g_{B}\right) \ll n_{0} \xi^{D}
$$

where $\xi \equiv \hbar / \sqrt{m_{B} g_{B} n_{0}}$ is the condensate healing length and $D=3$ for a 3D homogeneous bosonic bath [60]. Strictly speaking, even when the coupling $g_{I B}$ is stronger than this limit, the perturbation theory still qualitatively applies. For very strong interactions, however, the bath can not be properly described by Bogoliubov quasiparticles any more. The above perturbative method can also be used in a system where the impurities and BEC are both trapped by optical lattices [64]. 


\section{Atom-phonon coupling}

The impurity-BEC interaction of the Hamiltonian can be written as

$$
\begin{aligned}
\hat{H}_{\mathrm{int}} & =\sum_{\mathbf{q}} \sum_{\alpha, \beta} \sum_{i, j} \hbar \omega_{\mathbf{q}} M_{i, j ; \mathbf{q}}^{\alpha \beta} \hat{b}_{\mathbf{q}} \hat{a}_{i}^{\alpha \dagger} \hat{a}_{j}^{\beta}+h . c . \\
M_{i, j ; \mathbf{q}}^{\alpha \beta} & =\frac{g_{I B}}{\hbar \omega_{\mathbf{q}}} \sqrt{\frac{n_{0}}{\Omega}}\left(u_{\mathbf{q}}-v_{\mathbf{q}}\right) m_{i, j ; \mathbf{q}}^{\alpha \beta}
\end{aligned}
$$

with $\alpha, \beta$ indicating the impurity Bloch bands and $m_{i, j ; \mathbf{q}}^{\alpha \beta} \equiv \int d^{3} \mathbf{r} e^{i \mathbf{q} \cdot \mathbf{r}} W_{i}^{\alpha *}(\mathbf{r}) W_{j}^{\beta}(\mathbf{r})$. This term describes the impurity coupling to the phonon bath by creating or annihilating phonons. The non-local coupling terms with $i \neq j$ are highly suppressed due to the local form of the interaction which requires overlap between localized Wannier functions. For these reasons, the above integrals $m_{i, j ; \mathbf{q}}^{\alpha \beta}$ can be well approximated by $\delta_{i j} m_{\mathbf{q}}^{\alpha \beta} e^{i \mathbf{q} \cdot \mathbf{R}_{i}}$ such that the value depends only on $\alpha, \beta$ and $\mathbf{q}$. The dimensionless impurity-phonon coupling $M_{i ; \mathbf{q}}^{\alpha \beta}$ can also be written as $M_{\mathbf{q}}^{\alpha \beta} e^{i \mathbf{q} \cdot \mathbf{R}_{i}}$ with

$$
M_{\mathbf{q}}^{\alpha \beta} \equiv g_{I B} \sqrt{\frac{n_{0} \epsilon_{\mathbf{q}}}{\Omega\left(\hbar \omega_{\mathbf{q}}\right)^{3}}} m_{\mathbf{q}}^{\alpha \beta} .
$$

Note that the impurity-phonon coupling $M_{i, \mathbf{q}}^{\alpha \beta}$ obeys:

$$
\left(M_{i, \mathbf{q}}^{\alpha \beta}\right)^{*}=M_{i,-\mathbf{q}}^{\alpha \beta}
$$

The integral factors $m_{\mathbf{q}}^{\alpha \beta}$ take the form

$$
m_{\mathbf{q}}^{\alpha \beta}=\int d^{3} \mathbf{r} e^{i \mathbf{q} \cdot \mathbf{r}} \varphi_{x}^{\alpha *}(x) \varphi_{x}^{\beta}(x)\left|\phi^{0}(y)\right|^{2}\left|\phi^{0}(z)\right|^{2} .
$$

In a deep optical lattice, where the impurity Wannier functions in Eqs. (1-3) can be approximated as harmonic oscillator states, we can explicitly evaluate these factors:

$$
\begin{aligned}
& m_{\mathbf{q}}^{00}=e^{-\left(\sigma_{\perp}^{2} q_{\perp}^{2}+\sigma_{x}^{2} q_{x}^{2}\right) / 4}, \\
& m_{\mathbf{q}}^{01}=e^{-\left(\sigma_{\perp}^{2} q_{\perp}^{2}+\sigma_{x}^{2} q_{x}^{2}\right) / 4}\left(i q_{x} \sigma_{x} / \sqrt{2}\right)=m_{\mathbf{q}}^{10}, \\
& m_{\mathbf{q}}^{11}=e^{-\left(\sigma_{\perp}^{2} q_{\perp}^{2}+\sigma_{x}^{2} q_{x}^{2}\right) / 4}\left(1-q_{x}^{2} \sigma_{x}^{2} / 2\right),
\end{aligned}
$$

by using the identity for Hermite polynomial integrals [96]. Here $q_{\perp} \equiv \sqrt{q_{y}^{2}+q_{z}^{2}}$ indicates the transverse phonon momentum.

In order to describe polaronic effects resulting from the impurity-BEC coupling, we introduce a dimensionless coupling constant as in [73] and name it $\kappa$. This constant depends on the impurity-boson and boson-boson interactions $g_{I B}, g_{B B}$ and the condensate parameters $\xi$ and $m_{B}$ as

$$
\kappa \equiv \sqrt{\frac{g_{I B}^{2} m_{B}}{g_{B} \hbar^{2} \xi}}
$$

As described in [73], this constant is the ratio $\kappa=$ $E_{I B} / E_{\mathrm{ph}}$ between the characteristic impurity-boson interaction $E_{I B}=g_{I B} \sqrt{n_{0} \xi^{-3}}$ and the typical phonon energy $E_{\mathrm{ph}}=\hbar c / \xi$, where $c=\sqrt{g_{B} n_{0} / m_{B}}$ is the condensate speed of sound. In [62] an alternative dimensionless coupling constant is used as

$$
\alpha \equiv \frac{a_{I B}^{2}}{a_{B B} \xi},
$$

or equivalently $\alpha=4 \pi n_{0} a_{I B}^{2} \xi$. These two coupling constants are related by $\alpha=\left(\kappa \mu / m_{B}\right)^{2} / \pi$, where $\mu$ is the reduced mass. There are also other coupling constants used [59, 74], which are slightly different from $\alpha$ or $\kappa$. By tuning the impurity-boson scattering length $a_{I B}^{\text {eff }}$, the coupling constant $\kappa$ can be tuned continuously. However, the condition in eq. (10) requires that the coupling constant satisfies the relation

$$
\kappa \ll \kappa_{c} \equiv \frac{1}{2 \sqrt{\pi}} \sqrt{\frac{\xi}{a_{B}}}=\left(64 \pi^{3} a_{B}^{3} n_{0}\right)^{-\frac{1}{4}} .
$$

This upper limit of the coupling constant $\kappa_{c}$ depends only on the boson-boson scattering length $a_{B}$ and the condensate density $n_{0}$ instead of the specific mass ratio $m_{I} / m_{B}$. In a typical BEC system, this maximum coupling constant is relatively small, such as $\kappa_{c} \approx 2.4$ for ${ }^{87} \mathrm{Rb}$ with $a_{B}=100 a_{0}$ and $n_{0} \approx 10^{14} \mathrm{~cm}^{-3}$. In order to reach larger values of $\kappa_{c}$ in realistic systems, one need to reduce the condensate density $n_{0}$ or the Bose-Bose scattering length $a_{B}$

Finally the effective Hamiltonian with two-band Fröhlich impurity-phonon coupling is

$$
\begin{aligned}
\hat{H} & =-\sum_{\langle i, j\rangle} \sum_{\alpha} J^{\alpha} \hat{a}_{i}^{\alpha \dagger} \hat{a}_{j}^{\alpha}+\sum_{i} \sum_{\alpha} \varepsilon^{\alpha} \hat{n}_{i}^{\alpha} \\
& +\sum_{\mathbf{q}} \hbar \omega_{\mathbf{q}} \hat{b}_{\mathbf{q}}^{\dagger} \hat{b}_{\mathbf{q}} \\
& +\sum_{i, \mathbf{q}} \sum_{\alpha, \beta} \hbar \omega_{\mathbf{q}} M_{i, \mathbf{q}}^{\alpha \beta}\left(\hat{b}_{\mathbf{q}}+\hat{b}_{-\mathbf{q}}^{\dagger}\right) \hat{a}_{i}^{\alpha \dagger} \hat{a}_{i}^{\beta} .
\end{aligned}
$$

Here we used the relations in Eq. (14). This Hamiltonian describes a general two-band system with impurityphonon coupling, which can also be realized by other experimental setups such as hybrid atom-ion systems [87].

\section{VARIATIONAL TWO-BAND POLARON TRANSFORMATION}

\section{A. Transformation with exponential quadratic operators}

This two-band Hamiltonian in Eq. (19), with Fröhlichtype impurity-phonon coupling, can not be solved analytically even for the case of a single impurity. The goal of this paper is to find a simple but non-trivial variational method which can deal with the two-band system (19) 
in general. We choose the Lang-Firsov polaron transformation approach and generalize it to two-band system. This canonical transformation is exact and decouples the impurity-BEC interaction term in a new quasi-particle basis. In this basis, the kinetic part in Hamiltonian (19) contains the dynamics of quasiparticle and its interactions between the transformed phonon bath. We solve the coherent part of this transformed Hamiltonian with a variational treatment and take into account the remaining incoherent parts by a master equation.

Firstly we introduce the basic concept of the transformation for a single band before we extend it to the twoband case. When a single impurity moves in a lattice and couples to a phonon bath, there are exact solutions in both the weak and strong coupling limit [21]. When the impurity-phonon interaction is much weaker than the impurity kinetic energy, the impurity behaves as a free particle in a lattice. On the other hand, when the interaction is much larger than the kinetic part, the impurity will be tightly dressed by a "cloud" of phonons, forming a quasi-particle. The phonons are tied to the impurity such that the impurity cannot move on its own but must drag around a phonon cloud. This increases the effective mass of the quasiparticle. In the intermediate coupling region, the phonon dressing competes with the impurity dynamics. In order to describe this competition, a variational ground state can be used to connect between the weak and strong coupling limits [27, 69, 97-100]. This variational ansatz is equivalent to a canonical transformation $\tilde{H} \equiv e^{\hat{S}} \hat{H} e^{-\hat{S}}$ with $\hat{S} \equiv \sum_{i, \mathbf{q}} \Lambda_{i, \mathbf{q}}\left(\hat{b}_{-\mathbf{q}}^{\dagger}-\hat{b}_{\mathbf{q}}\right) \hat{n}_{i}$ where $\Lambda_{i, \mathbf{q}}$ are the variational parameters. The transformed Hamiltonian $\tilde{H}$ still cannot be solved analytically, but can be separated into a coherent part $\langle\tilde{H}\rangle_{T}$ and an incoherent part $\tilde{H}_{\text {inc }} \equiv \tilde{H}-\langle\tilde{H}\rangle_{T}$ where $\langle\cdots\rangle_{T}$ indicates a thermal average over the phonon bath. The coherent part, which is decoupled from the phonon bath, is of the form of an extended (polaronic) Hubbard model. The incoherent part describes the residual coupling between polaron quasi-particle and phonon bath. Compared to the initial "bare" impurity-phonon coupling, this incoherent part is significantly reduced by the polaron transformation. We first focus on the coherent part and neglect the incoherent terms. The variational parameters $\Lambda_{i, \mathbf{q}}$ are determined by minimizing the coherent Hamiltonian energy and approach $\Lambda_{i, \mathbf{q}}=M_{i, \mathbf{q}}$ in the strong coupling limit. Finally, the residual incoherent part can be included by a perturbative approach such as the Lindblad master equation.

In order to find a suitable variational transformation for the two-band system, we modify the Lang-Firsov polaron transformation $\tilde{H}=e^{\hat{S}} H e^{-\hat{S}}$ by extending the impurity-phonon interaction to the two-band form. It takes the form:

$$
\hat{S} \equiv\left[\sum_{i, \mathbf{q}} \sum_{\alpha, \beta} \Lambda_{i, \mathbf{q}}^{\alpha \beta}\left(\hat{b}_{-\mathbf{q}}^{\dagger}-\hat{b}_{\mathbf{q}}\right) \hat{a}_{i}^{\alpha \dagger} \hat{a}_{i}^{\beta}\right],
$$

with $\Lambda_{i, \mathbf{q}}^{\alpha \beta} \equiv \Lambda_{\mathbf{q}}^{\alpha \beta} e^{i \mathbf{q} \cdot \mathbf{R}_{i}}$. A similar method was also applied by Sibley and Munn in [101-104] and Stojanović et al. in [99] for a single-band system with non-local impurity-phonon coupling. Our initial guess for the variational parameters is the coupling element itself, $M_{\mathbf{q}}^{\alpha \beta}$, and we constrain the variational parameters to obey the same symmetry properties as $M_{\mathbf{q}}^{\alpha \beta}$, namely that of Eq. (14). By using the Baker-Campbell-Hausdorff formula, $e^{\hat{S}} \hat{A} e^{-\hat{S}}=\hat{A}+[\hat{S}, \hat{A}]+\frac{1}{2 !}[\hat{S},[\hat{S}, \hat{A}]]+\cdots$, we can derive the transformed Hamiltonian with exponential quadratic operators as outlined in Appendix A. For convenience, these expressions can be written in $2 \times 2$ matrix form via

$$
\Lambda_{\mathbf{q}} \equiv\left(\begin{array}{cc}
\Lambda_{\mathbf{q}}^{00} & \Lambda_{\mathbf{q}}^{01} \\
\Lambda_{\mathbf{q}}^{10} & \Lambda_{\mathbf{q}}^{11}
\end{array}\right) ; \hat{\mathbf{b}}_{\mathbf{q}} \equiv\left(\begin{array}{cc}
\hat{b}_{\mathbf{q}} & 0 \\
0 & \hat{b}_{\mathbf{q}}
\end{array}\right)
$$

and

$$
\mathbf{M}_{i, \mathbf{q}} \equiv\left(\begin{array}{cc}
M_{\mathbf{q}}^{00} & M_{\mathbf{q}}^{01} \\
M_{\mathbf{q}}^{10} & M_{\mathbf{q}}^{11}
\end{array}\right) e^{i \mathbf{q} \cdot \mathbf{R}_{i}}
$$

After the transformation, the impurity annihilation and creation operators can be expressed through

$$
e^{\hat{S}} \hat{a}_{i}^{\alpha} e^{-\hat{S}}=\sum_{\beta}\left(\hat{\mathbf{X}}_{i}\right)_{\alpha \beta} \hat{a}_{i}^{\beta},
$$

and similarly for the creation operators. The matrix operators $\hat{\mathbf{X}}_{i}$ are found to be

$$
\hat{\mathbf{X}}_{i} \equiv e^{-\sum_{\mathbf{q}} \boldsymbol{\Lambda}_{\mathbf{q}} e^{i \mathbf{q} \cdot \mathbf{R}_{i}}\left(\hat{\mathbf{b}}_{-\mathbf{q}}^{\dagger}-\hat{\mathbf{b}}_{\mathbf{q}}\right)}
$$

In the same fashion as was described above for the single band system, this canonical transformation is equivalent to defining a new quasi-particle, which represents an impurity dressed by the phonon cloud forming a polaron. This transformation also shifts the equilibrium position of the phonon bath by

$$
e^{\hat{S}} \hat{b}_{\mathbf{q}} e^{-\hat{S}}=\hat{b}_{\mathbf{q}}+\sum_{i, \alpha, \beta}\left(\hat{\mathbf{X}}_{i}^{\dagger} \hat{\mathbf{b}}_{\mathbf{q}} \hat{\mathbf{X}}_{i}-\hat{\mathbf{b}}_{\mathbf{q}}\right)_{\alpha \beta} \hat{a}_{i}^{\alpha \dagger} \hat{a}_{i}^{\beta},
$$

but it does not modify the phonon dispersion relation. In Appendix A, we show the derivations for Eq. (21-23). The transformed polaronic Hamiltonian can be written as: 


$$
\begin{aligned}
\tilde{H} & =-\sum_{\langle i, j\rangle} \sum_{\alpha \beta}\left(\hat{\mathbf{X}}_{i}^{\dagger} \mathbf{J} \hat{\mathbf{X}}_{j}\right)_{\alpha \beta} \hat{a}_{i}^{\alpha \dagger} \hat{a}_{j}^{\beta}+\sum_{i} \sum_{\alpha \beta}\left(\hat{\mathbf{X}}_{i}^{\dagger} \varepsilon \hat{\mathbf{X}}_{i}\right)_{\alpha \beta} \hat{a}_{i}^{\alpha \dagger} \hat{a}_{i}^{\beta}+\sum_{\mathbf{q}} \hbar \omega_{\mathbf{q}} \hat{b}_{\mathbf{q}}^{\dagger} \hat{b}_{\mathbf{q}} \\
& +\sum_{i, \alpha, \beta} \sum_{\mathbf{q}} \hbar \omega_{\mathbf{q}}\left[\left(\hat{\mathbf{X}}_{i}^{\dagger} \hat{\mathbf{b}}_{\mathbf{q}}^{\dagger} \mathbf{M}_{i, \mathbf{q}}^{\dagger} \hat{\mathbf{X}}_{i}\right)+\left(\hat{\mathbf{X}}_{i}^{\dagger} \mathbf{M}_{i, \mathbf{q}} \hat{\mathbf{b}}_{\mathbf{q}} \hat{\mathbf{X}}_{i}\right)+\left(\hat{\mathbf{X}}_{i}^{\dagger} \hat{\mathbf{b}}_{\mathbf{q}}^{\dagger} \hat{\mathbf{b}}_{\mathbf{q}} \hat{\mathbf{X}}_{i}\right)-\hat{\mathbf{b}}_{\mathbf{q}}^{\dagger} \hat{\mathbf{b}}_{\mathbf{q}}\right]_{\alpha \beta} \hat{a}_{i}^{\alpha \dagger} \hat{a}_{i}^{\beta} \\
& +\sum_{i, \alpha, \beta} \sum_{j, \alpha^{\prime}, \beta^{\prime}} \sum_{\mathbf{q}} \frac{\hbar \omega_{\mathbf{q}}}{2}\left[\left(\hat{\mathbf{X}}_{i}^{\dagger} \hat{\mathbf{b}}_{\mathbf{q}}^{\dagger} \hat{\mathbf{X}}_{i}-\hat{\mathbf{b}}_{\mathbf{q}}^{\dagger}\right)_{\alpha \beta}\left(2 \hat{\mathbf{X}}_{j}^{\dagger} \mathbf{M}_{j, \mathbf{q}}^{\dagger} \hat{\mathbf{X}}_{j}+\hat{\mathbf{X}}_{j}^{\dagger} \hat{\mathbf{b}}_{\mathbf{q}} \hat{\mathbf{X}}_{j}-\hat{\mathbf{b}}_{\mathbf{q}}\right)_{\alpha^{\prime} \beta^{\prime}}+h . c .\right] \hat{a}_{i}^{\alpha \dagger} \hat{a}_{j}^{\alpha^{\prime} \dagger} \hat{a}_{j}^{\beta^{\prime}} \hat{a}_{i}^{\beta},
\end{aligned}
$$

where $\mathbf{J}$ and $\varepsilon$ is the (diagonal) matrix form of $J^{\alpha}$ and $\varepsilon^{\alpha}$. Here we combine all single impurity contributions in the second line. The last term, which is zero when only a single impurity is considered, describes induced polaronpolaron interactions due to the coupling with the phonon bath. Compared to the original Hamiltonian in Eq. (19), the hopping and on-site energy have been modified. As for the single band system, this Hamiltonian contains all interactions exactly and is hard to solve analytically. Motivated by the single-band system, we separate the Hamiltonian into coherent and incoherent parts, $\langle\tilde{H}\rangle_{T}$ and $\tilde{H}-\langle\tilde{H}\rangle_{T}$ respectively. The explicit form of the coherent terms, which conserve the number of phonons, is determined in Appendix A. In Eqs. (A17, A18, A25, A28) we calculate all possible coherent terms in Eq. (24). After determining the variational parameters $\Lambda_{\mathbf{q}}$ by minimizing the free energy of the coherent part, we treat the residual polaron-bath coupling in the incoherent part as a perturbation and solve it by a Lindblad master equation.

\section{B. Diagonal transformation matrix}

In Appendix A, we calculate the coherent part $\langle\tilde{H}\rangle_{T}$ by averaging over the phonon bath and assuming it is thermal. In contrast to the single-band case, these calculations are demanding when both intra- and inter-band phonon couplings are included. In order to determine the variational parameters $\boldsymbol{\Lambda}_{\mathbf{q}}$, we finally need to minimize the free energy of whole coherent Hamiltonian. Until now, we did not make any assumptions for our variational parameters $\boldsymbol{\Lambda}_{\mathbf{q}}$ except for the symmetry relations in Eq. (14).

Unfortunately, the general result of the transformed Hamiltonian in Eq. (24) and its corresponding coherent part are still quite complicated. It can be further simplified by making some approximations suitable to our specific system. Due to conservation of energy, the phononinduced inter-band dynamics requires the phonon energy to match the impurity band gap, i.e. $\hbar \omega_{\mathbf{q}} \approx \varepsilon^{\Delta}$. This energy scale involves a phonon with particle-like dispersion and momentum $|\mathbf{q}| \approx \sqrt{2 m_{B} \varepsilon^{\Delta}} / \hbar$ significantly far from zero. The inter-band coupling $M_{\mathbf{q}}^{01}$ for this large phonon momentum is highly reduced due to the Gaussian decay of $m_{\mathbf{q}}^{01}$ in Eq. (15). On the other hand, intra- band dynamics requires a phonon energy $\hbar \omega_{\mathbf{q}} \approx J^{0} ; J^{1}$ with phonon-like dispersion and small momentum $|\mathbf{q}| \approx$ $J^{\alpha} \sqrt{m_{B} /\left(g_{B} n_{0}\right)} / \hbar=J^{\alpha} /(\hbar c)$. The intra-band coupling $M_{\mathbf{q}}^{\alpha \alpha}$ is not reduced too much at this smaller momentum. In the polaron transformation, the parameters $\Lambda_{\mathbf{q}}^{\alpha \beta}$ reflect the dressing of the impurity by phonons and are closely related to $M_{\mathbf{q}}^{\alpha \beta}$. For this reason, we treat the inter-band coupling as small and approximate the matrices $\boldsymbol{\Lambda}_{\mathbf{q}}$ as diagonal:

$$
\boldsymbol{\Lambda}_{\mathbf{q}}=\left[\begin{array}{cc}
\lambda_{\mathbf{q}}^{0} M_{\mathbf{q}}^{00} & 0 \\
0 & \lambda_{\mathbf{q}}^{1} M_{\mathbf{q}}^{11}
\end{array}\right]
$$

with variational parameters $\lambda_{\mathbf{q}}^{0}$ and $\lambda_{\mathbf{q}}^{1}$. Since the intraband couplings $M_{\mathbf{q}}^{\alpha \alpha}$ are purely real numbers in our system, we assume $\lambda_{\mathbf{q}}^{\alpha}$ are also real numbers. After the transformation with these diagonal matrices $\boldsymbol{\Lambda}_{\mathbf{q}}$, we have then decoupled the intra-band impurity-phonon coupling and leave the (relatively) small inter-band coupling in the new polaronic two-band Hamiltonian. The coherent part, with phonons eliminated by thermal averaging, is a many-body Hamiltonian:

$$
\begin{aligned}
\langle\tilde{H}\rangle_{T}= & -\sum_{\langle i, j\rangle, \alpha} J_{\mathrm{P}}^{\alpha} \hat{a}_{i}^{\alpha \dagger} \hat{a}_{j}^{\alpha}+\sum_{i, \alpha} \varepsilon_{P}^{\alpha} \hat{n}_{i}^{\alpha}+\sum_{\mathbf{q}} \hbar \omega_{\mathbf{q}}\left\langle\hat{b}_{\mathbf{q}}^{\dagger} \hat{b}_{\mathbf{q}}\right\rangle_{T} \\
& +\hat{V}_{\mathrm{P}}
\end{aligned}
$$

with renormalized polaronic hopping terms $J_{\mathrm{P}}^{\alpha}$ and onsite energy $\varepsilon_{\mathrm{P}}^{\alpha}$ including the polaron energy shift:

$$
\begin{aligned}
& J_{\mathrm{P}}^{\alpha} \equiv J^{\alpha}\left\langle\left(\hat{\mathbf{X}}_{i}\right)_{\alpha \alpha}^{\dagger}\left(\hat{\mathbf{X}}_{j}\right)_{\alpha \alpha}\right\rangle_{T}, \\
& \varepsilon_{\mathrm{P}}^{\alpha} \equiv \varepsilon^{\alpha}-\sum_{\mathbf{q}} \hbar \omega_{\mathbf{q}}\left(\Lambda_{\mathbf{q}}^{\alpha \alpha}\right)\left(2 M_{\mathbf{q}}^{\alpha \alpha}-\Lambda_{\mathbf{q}}^{\alpha \alpha}\right)^{*} .
\end{aligned}
$$

In the above formula, we use the fact that operators $\hat{\mathbf{X}}_{i}$ have only diagonal terms. There are also induced interactions $V_{\mathrm{P}}$ between multiple polarons, which can lead to strong correlations in the system and will be discussed in the next section. In the above calculations, we need to sum over all possible phonon momenta $\mathbf{q}$. In the thermodynamic limit of the phonon bath, we use the relation $\sum_{\mathbf{q}} \rightarrow \frac{\Omega}{(2 \pi)^{D}} \int d \mathbf{q}$ with quantization volume $\Omega$ for the phonons, and write this explicitly in cylindrical coordinates: $\frac{\Omega}{(2 \pi)^{3}} \int d q_{\perp} \int d q_{x} 2 \pi q_{\perp}$. 
For the polaronic intra-band hopping $J_{\mathrm{P}}^{\alpha}$, we only need to calculate nearest-neighbor terms with $j=i \pm 1$ regardless of the specific value of $i$. By noting that

$$
\left\langle\left(\hat{\mathbf{X}}_{i}\right)_{\alpha \alpha}^{\dagger}\left(\hat{\mathbf{X}}_{j}\right)_{\beta \beta}\right\rangle_{T}=e^{-\sum_{\mathbf{q}}\left(N_{\mathbf{q}}+1 / 2\right)\left|\Lambda_{i, \mathbf{q}}^{\alpha \alpha}-\Lambda_{j, \mathbf{q}}^{\beta \beta}\right|^{2}}
$$

where $N_{\mathbf{q}} \equiv\left(\exp \left(\hbar \omega_{\mathbf{q}} / k_{\mathrm{B}} T\right)-1\right)^{-1}$ is the thermally averaged phonon occupation number, we define polaronic renormalization factors

$$
S_{T}^{\alpha} \equiv \sum_{\mathbf{q}}\left(2 N_{\mathbf{q}}+1\right)\left[1-\cos \left(q_{x} \cdot d\right)\right]\left|\Lambda_{\mathbf{q}}^{\alpha \alpha}\right|^{2}
$$

for each band and thus $J_{\mathrm{P}}^{\alpha}=J^{\alpha} \exp \left(-S_{T}^{\alpha}\right)$.

We would like to note that the choice of diagonal transformation matrices $\boldsymbol{\Lambda}_{\mathbf{q}}$ works well when the inter-band coupling is less important that the intra-band coupling. By choosing diagonal matrices, we treat each of the two bands independently. This approach is similar as the authors in Ref. [60, 61] did for single band problem, except a variational treatment was used. On the other hand, when the off-diagonal terms in the coupling matrix $\mathbf{M}_{\mathbf{q}}$ are comparable or larger than the diagonal terms, one must instead deal with the general transformed Hamiltonian in Eq. (24). For completeness, we describe a more general approximate technique in Appendix $A$, which may be useful for other types of systems. We intend to address the approximation introduced in this section more explicitly and quantitatively in a future publication.

\section{COHERENT POLARON DYNAMICS AND INTERACTIONS}

\section{A. Single polaron band structures}

In the previous section, we derived a general form of the two-band polaron transformation and calculated the resulting coherent part of the Hamiltonian in Eq. (25). For the system with a single polaron, there are only intraband terms in the coherent Hamiltonian (25). The single impurity is dressed by a coherent phonon cloud in each band. The residual inter-band polaron-bath coupling will appear only in the incoherent Hamiltonian. It is easy to diagonalize the single-polaron coherent part in the momentum representation and minimize the free energy $F \equiv-k_{\mathrm{B}} T \ln \sum_{\alpha, k} \exp \left(-E_{k}^{\alpha} / k_{\mathrm{B}} T\right)$ for this twoband system, with polaron dispersion:

$$
E_{k}^{\alpha} \equiv 2 J_{\mathrm{P}}^{\alpha} \cos (k \cdot d)+\varepsilon_{\mathrm{P}}^{\alpha}
$$

Here $k$ is quasi-momentum in longitudinal direction. These variational parameters, which are real numbers, can then be determined by the self-consistent equations:

$$
\lambda_{\mathbf{q}}^{\alpha}=\frac{\sum_{k, \alpha^{\prime}} \exp \left(-E_{k}^{\alpha^{\prime}} / k_{\mathrm{B}} T\right)}{\sum_{k, \alpha^{\prime}}\left[1-2 J_{\mathrm{P}}^{\alpha} f_{\mathbf{q}} \cos (k \cdot d) / \omega_{\mathbf{q}}\right] \exp \left(-E_{k}^{\alpha^{\prime}} / k_{\mathrm{B}} T\right)}
$$

with $f_{\mathbf{q}} \equiv\left(2 N_{\mathbf{q}}+1\right)\left[1-\cos \left(q_{x} d\right)\right]$.

Before numerically calculating the variational parameters $\lambda_{\mathbf{q}}^{\alpha}$, we firstly discuss some properties of this selfconsistent equation. Considering a simplified model with momentum-independent variational parameters $\lambda$ in a single band system, we choose to minimize only the ground state energy. The self-consistent equation (30) will be modified as

$$
\lambda=\left[1+2|J| \frac{\sum_{\mathbf{q}} f_{\mathbf{q}}\left|M_{\mathbf{q}}\right|^{2}}{\sum_{\mathbf{q}} \hbar \omega_{\mathbf{q}}\left|M_{\mathbf{q}}\right|^{2}} e^{-\lambda^{2} \sum_{\mathbf{q}} f_{\mathbf{q}}\left|M_{\mathbf{q}}\right|^{2}}\right]^{-1}
$$

From the analysis in $[99,100]$, this equation has two locally stable solutions $\lambda_{-}, \lambda_{+}$once the adiabatic regime is achieved when

$$
2|J| \frac{\sum_{\mathbf{q}} f_{\mathbf{q}}\left|M_{\mathbf{q}}\right|^{2}}{\sum_{\mathbf{q}} \hbar \omega_{\mathbf{q}}\left|M_{\mathbf{q}}\right|^{2}}>\frac{e^{3 / 2}}{2} .
$$

These two solutions $\lambda_{-}, \lambda_{+}$, corresponding to two local minima of ground state energy, indicate the impurity is respectively loosely or tightly dressed by phonons. At a critical impurity-phonon coupling with $\sum_{\mathbf{q}} f_{\mathbf{q}}\left|M_{\mathbf{q}}\right|^{2}=$ $27 / 8$, when the two minima become equal, the lowest energy state solution abruptly switches from $\lambda_{-}$to $\lambda_{+}$, indicating a first-order polaronic transition. On the other hand, the solution of $\lambda$ is a smooth and continuous crossover when the adiabatic condition is broken with small value of $|J|$. This transition-crossover behavior also appears later when solving the self-consistent equation (30) numerically, although we have considered a much more simplified model here. Strictly speaking, this sharp polaronic transition in the adiabatic regime is due to the mean-field approximation by thermal averaging of the phonon degrees of freedom. This drawback could be improved if we were to treat the incoherent dynamics properly, by taking into account fluctuations or using a master equation method.

We now compare the variational parameters $\lambda_{\mathbf{q}}^{\alpha}=$ $\Lambda_{\mathbf{q}}^{\alpha \alpha} / M_{\mathbf{q}}^{\alpha \alpha}$ from Eq. (30) and polaron dressing effects for different system parameters. In Fig. 2(a) we show the coupling factor $\lambda_{\mathbf{q}}^{\alpha}$ in each band for the temperatures $k_{\mathrm{B}} T=0, E_{R}, 2 E_{R}$, where it can be seen that the variational parameters $\lambda_{\mathbf{q}}^{\alpha}$ are always smaller than 1. This shows the competition between intra-band dynamics and phonon dressing effects. These variational parameters are also different for the two bands, since the polaron is dressed differently in each band. We have focused on the results for momentum in the longitudinal direction with $\mathbf{q}=\left(q_{x}, 0,0\right)$ and find that the low-momentum phonons are less dressed at higher temperatures $k_{\mathrm{B}} T \approx E_{R} \gg J^{\alpha}$. Here $E_{R}$ corresponds to a temperature of about $65 \mathrm{nK}$ for a ${ }^{133} \mathrm{Cs}$ impurity trapped by lasers with wavelength $1064 \mathrm{~nm}$. In Fig. 2(b, c) we also show the the factors $\lambda^{\alpha} \equiv \sum_{\mathbf{q}} \Lambda_{\mathbf{q}}^{\alpha \alpha} / \sum_{\mathbf{q}} M_{\mathbf{q}}^{\alpha \alpha}$. These factors $\lambda^{\alpha}$ thus indicate the differences between our initial guess for the variational parameters $\Lambda_{\mathbf{q}}^{\alpha \alpha}=M_{\mathbf{q}}^{\alpha \alpha}$, as is sometimes used for the transformation, and the full minimization of free energy for the variational parameters. As shown in Fig. 2, 
(a)

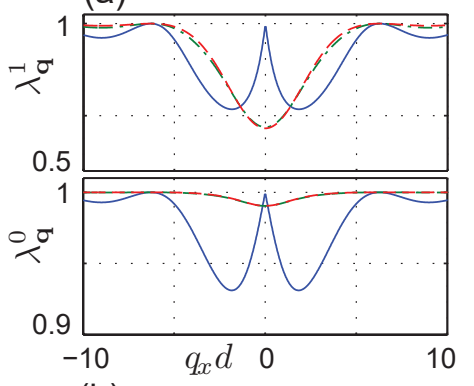

(b)

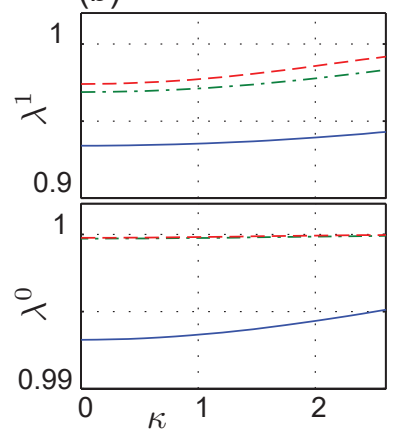

(c)

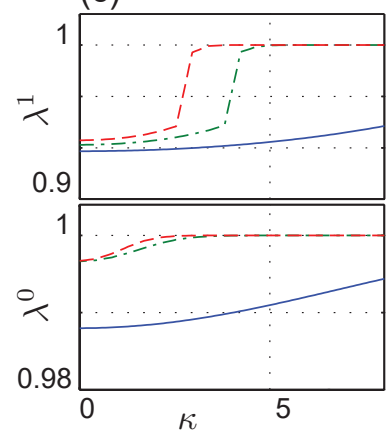

$E_{k}^{\alpha}$, renormalization of intra-band hopping $J_{\mathrm{P}}^{\alpha} / J^{\alpha}$, polaron effective mass $m_{\mathrm{P}}^{\alpha} / m_{0}^{\alpha}$ and renormalization factor $S_{T}^{\alpha}$ for each band at different temperatures.

Due to phonon dressing effects, the polaronic band gap $\varepsilon_{\mathrm{P}}^{\Delta} \equiv \varepsilon_{\mathrm{P}}^{1}-\varepsilon_{\mathrm{P}}^{0}$ is also increased. This will affect the interband relaxation dynamics. In Fig. 4 we show the onsite polaron energy and band gap renormalization versus impurity-BEC coupling constant. In both Fig. 3(a) and Fig. 4(a), without loss of generality, we set the initial lower band on-site energy $\varepsilon^{0}$ to zero. As shown in Fig. 4(b), the band gap renormalization is almost temperature independent. These quantities are only slightly affected by temperature due to different variational transformation matrices $\Lambda_{\mathbf{q}}$, as predicted in Eq. (26). Although the band gap is not significantly changed, this renormalization effect is important for inter-band resonance conditions, which are required for Landau-Zener tunneling to take place in a tilted lattice $[105,106]$.

\section{B. Effective interactions between polarons}

Here we briefly discuss interactions between polarons,

Figure 2: (a) Variational parameters $\lambda_{\mathbf{q}}^{\alpha}$ with $\mathbf{q}=\left(q_{x}, 0,0\right)$ and $\kappa=1$ in higher band (upper) and lower band (lower) at different temperatures $k_{\mathrm{B}} T=0, E_{R}, 2 E_{R}$. (b)(c) Factors $\lambda^{\alpha} \equiv \sum_{\mathbf{q}} \Lambda_{\mathbf{q}}^{\alpha \alpha} / \sum_{\mathbf{q}} M_{\mathbf{q}}^{\alpha \alpha}$ in each band at different temperatures. Maximum coupling constant $\kappa_{c}=2.4$ for condensate density $n_{0}=1 \times 10^{14} \mathrm{~cm}^{-3}$ (b) and $\kappa_{c}=7.6$ for condensate density $n_{0}=0.01 \times 10^{14} \mathrm{~cm}^{-3}(\mathrm{c})$. Other parameters are: $m_{I}=133, m_{B}=87 ; V_{I}^{x}=9 E_{R}, V_{I}^{\perp}=25 V_{I}^{x}$ and $a_{B}=100 a_{0}$.

although these factors are different for each band and different temperatures, they always approach $\lambda^{\alpha}=1$ in the limit of strong interaction. In order to reach larger values of the coupling constant, in Fig. 2(c) we show results with $\kappa_{c}=7.6$ by assuming condensate density $n_{0}=0.01 \times 10^{14} \mathrm{~cm}^{-3}$. In the upper plot of Fig. 2(c), we notice that the parameter $\lambda$ shows a polaronic transition in the adiabatic regime when the condition Eq. (32) is satisfied at finite temperature. On the other hand, $\lambda$ shows a smooth crossover behavior in the non-adiabatic regime at zero temperature.

In the coherent part of the Hamiltonian Eq. (25), the single polaron band structure is modified by phonon dressing effects in Eq. (26). Effectively, the polaron is trapped in a deeper lattice, with larger mass. The effective mass of a single polaron (at $k_{0}=0$ ) in an optical lattice can be defined as

$$
m_{\mathrm{P}}^{\alpha}\left(k_{0}\right) \equiv \hbar^{2}\left(\left.\frac{\partial^{2} E_{k}^{\alpha}}{\partial k^{2}}\right|_{k_{0}}\right)^{-1}=\frac{\hbar^{2}}{2 J_{\mathrm{P}}^{\alpha}} .
$$

If the impurity-BEC coupling $g_{I B}$ increases, the polaron effective mass will increase exponentially as $m_{\mathrm{P}}^{\alpha}=$ $m_{0}^{\alpha} \exp \left(S_{T}^{\alpha}\right)$, with $m_{0}^{\alpha}$ indicating the impurity effective mass at $\kappa=0$. In Fig. 3 we compare energy spectrum

by considering the additional interactions between impurities beyond the original Hamiltonian (19), which are given by

$$
\hat{V}_{0}=\sum_{i} \sum_{\alpha \beta} U^{\alpha \beta} \hat{n}_{i}^{\alpha}\left(\hat{n}_{i}^{\beta}-\delta_{\alpha \beta}\right)
$$

with the impurity on-site intra-/inter-band Hubbardtype interaction $U^{\alpha \beta}$. After the polaron transformation, the coherent Hamiltonian Eq. (25) for the multi-polaron system contains effective interactions $\hat{V}_{\mathrm{P}}$ with both intraand inter-band terms. In principle, the variational parameters $\lambda_{\mathbf{q}}$ should be determined by minimizing the total free energy. This is hard for the many-impurity system. Here we assume that the polaron parameters are not modified from the single polaron case, due to the low density of impurities in our dilute system. The effective interaction terms $\hat{V}_{\mathrm{P}}$ in Hamiltonian Eq. (25) take the form:

$$
\begin{aligned}
\hat{V}_{\mathrm{P}} & =\sum_{i} \sum_{\alpha \beta}\left(U^{\alpha \beta}+V_{i i}^{\alpha \beta}\right) \hat{n}_{i}^{\alpha}\left(\hat{n}_{i}^{\beta}-\delta_{\alpha \beta}\right) \\
& +\sum_{i \neq j} \sum_{\alpha \beta}\left(V_{i, j}^{\alpha \beta} \hat{n}_{i}^{\alpha} \hat{n}_{j}^{\beta}+\sum_{\beta^{\prime} \neq \beta} V_{i, j}^{\alpha ; \beta \beta^{\prime}} \hat{n}_{i}^{\alpha} \hat{a}_{j}^{\beta \dagger} \hat{a}_{j}^{\beta^{\prime}}\right)
\end{aligned}
$$

with long-range bath induced interaction terms:

$$
\begin{aligned}
V_{i, j}^{\alpha \beta} \equiv- & \sum_{\mathbf{q}} \hbar \omega_{\mathbf{q}} \cos \left[q_{x}(i-j) d\right] \\
& \times\left(\Lambda_{\mathbf{q}}^{\alpha \alpha}\right)\left(2 M_{\mathbf{q}}^{\beta \beta}-\Lambda_{\mathbf{q}}^{\beta \beta}\right)
\end{aligned}
$$


(a)

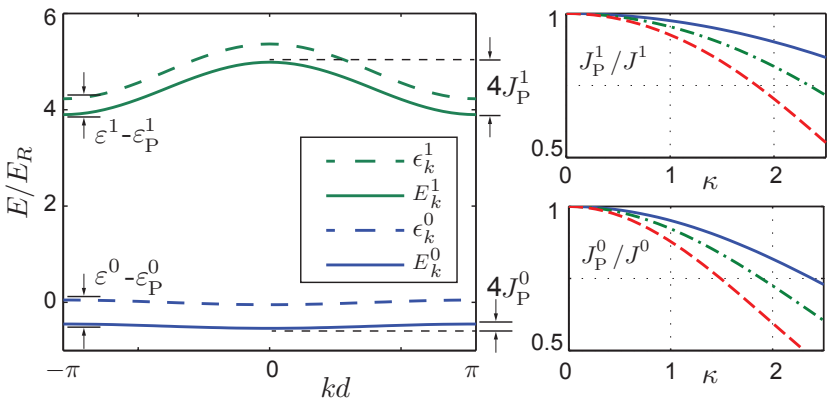

(c)

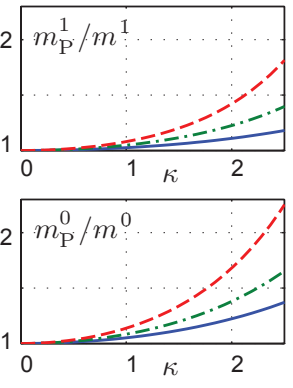

(d)

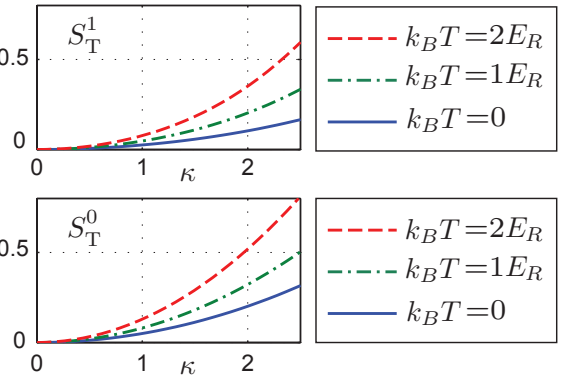

Figure 3: (a) Dispersion relation for bare impurity $\epsilon_{k}^{\alpha}$ and for polaron $E_{k}^{\alpha}$ with $\kappa=\kappa_{c}$; (b) renormalized hopping $J_{\mathrm{P}}^{\alpha} / J^{\alpha}$; (c) polaron effective mass $m_{\mathrm{P}}^{\alpha} / m_{0}^{\alpha} ;$ (d) renormalization factor $S_{T}^{\alpha}$ for each band at different temperatures $k_{\mathrm{B}} T=0 ; E_{R} ; 2 E_{R}$. Other parameters are $m_{I}=133, m_{B}=87 ; V_{I}^{x}=9 E_{R}, V_{I}^{\perp}=25 V_{I}^{x} ; a_{B}=100 a_{0}, n_{0}=1 \times 10^{14} \mathrm{~cm}^{-3}$ and $\kappa_{c}=2.4$.

(a)

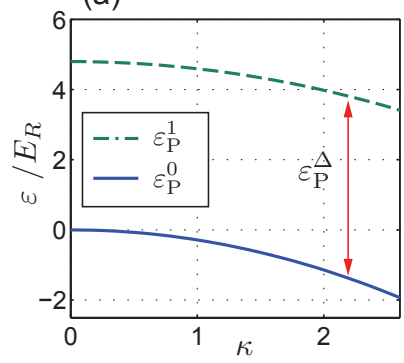

(b)

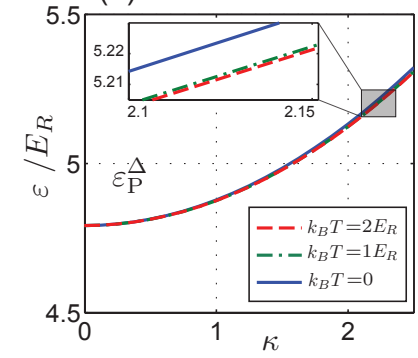

Figure 4: (a) On-site energy $\varepsilon_{\mathrm{P}}^{\alpha}$ including polaron energy shift at zero temperature; (b) renormalized band gap $\varepsilon_{\mathrm{P}}^{\Delta} \equiv \varepsilon_{\mathrm{P}}^{1}-\varepsilon_{\mathrm{P}}^{0}$ at different temperatures $k_{\mathrm{B}} T=0 ; E_{R} ; 2 E_{R}$. The energy renormalization is almost temperature-independent. Other parameters are $m_{I}=133, m_{B}=87 ; V_{I}^{x}=9 E_{R}, V_{I}^{\perp}=25 V_{I}^{x}$; $a_{B}=100 a_{0}, n_{0}=1 \times 10^{14} \mathrm{~cm}^{-3}$ and $\kappa_{c}=2.4$.

and

$$
\begin{aligned}
V_{i, j}^{\alpha ; \beta \beta^{\prime}} \equiv & -\sum_{\mathbf{q}} \hbar \omega_{\mathbf{q}} \sin \left[q_{x}(i-j) d\right] \\
& \times\left(\Lambda_{\mathbf{q}}^{\alpha \alpha}\right)\left(2 i \cdot M_{\mathbf{q}}^{\beta \beta^{\prime}}\right)\left\langle\hat{K}_{j, j}^{\beta \beta^{\prime}}\right\rangle_{T},
\end{aligned}
$$

with

$$
\hat{K}_{i, j}^{\alpha \beta} \equiv\left(\hat{\mathbf{X}}_{i}\right)_{\alpha \alpha}^{\dagger}\left(\hat{\mathbf{X}}_{j}\right)_{\beta \beta}=e^{-\sum_{\mathbf{q}}\left(\Lambda_{i, \mathbf{q}}^{\alpha \alpha}-\Lambda_{j, \mathbf{q}}^{\beta \beta}\right)^{*} \hat{b}_{\mathbf{q}}^{\dagger}-h . c .} .
$$

As it was shown in [60, 107], these induced densitydensity interactions $V_{i, j}^{\alpha \beta}$ are always attractive with $V_{i, j}^{\alpha \beta}<0$ and take the form of Yukawa-type interactions. The first line in Eq. (35) contains on-site effective interactions between polarons, also within different bands. Due to these attractive interactions $V_{i, j}^{\alpha \beta}$, the final on-site interactions $\left(U^{\alpha \beta}+V_{i i}^{\alpha \beta}\right)$ must be repulsive for bosonic impurities in order to keep the system stable. The second part in Eq. (35) describes the longrange part of these induced interactions. There are also

(a)

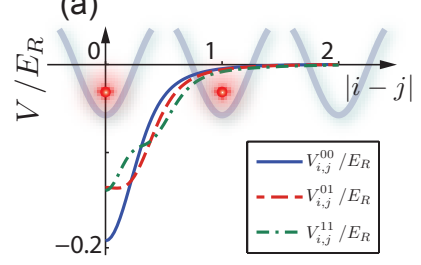

(c)
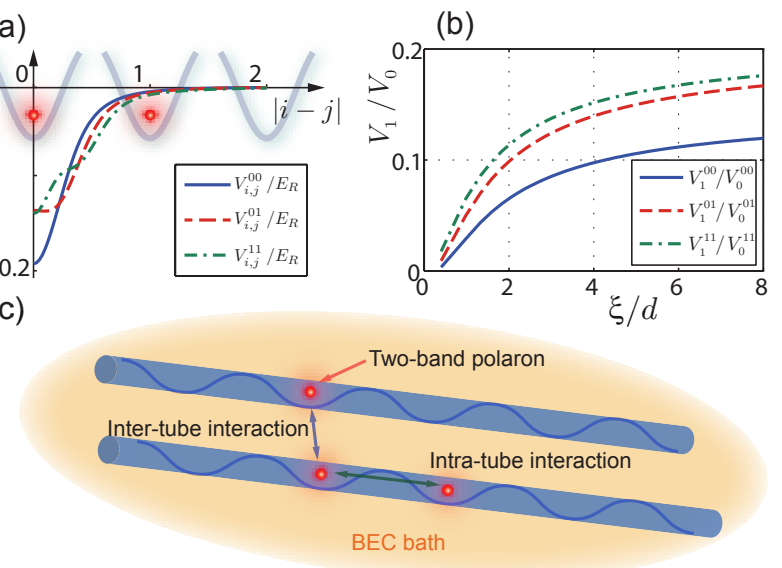

Figure 5: (a) Induced density-density interactions $V_{i, j}^{\alpha \beta}$ between different band at $\kappa=1$. (b) Ratio between induced on-site and nearest neighbor interaction terms. The healing length $\xi$ is increased by reducing the condensate density $n_{0}$ from $1 \times 10^{14} \mathrm{~cm}^{-3}$ to $0.01 \times 10^{14} \mathrm{~cm}^{-3}$ while keeping the coupling constant $\kappa=0.3 \kappa_{c}$. (c) Effective polaronic interactions in a multiple tube setup with two Bloch bands. Other parameters are $m_{I}=133, m_{B}=87 ; V_{I}^{x}=9 E_{R}, V_{I}^{\perp}=25 V_{I}^{x}$ and $a_{B}=100 a_{0} ; n_{0}=1 \times 10^{14} \mathrm{~cm}^{-3}$.

density-induced inter-band transitions, which are due to the inter-band polaron-bath coupling.

In Fig. 5(a) we show the induced density-density interactions $V_{i, j}^{\alpha \beta}$ versus distance $|i-j|$ between polarons between each band. Since these interactions are induced by the impurity-BEC coupling, the long-range behavior is related to the condensate healing length $\xi$. Here we assume we can obtain large values for $\xi$ by tuning the Bose-Bose scattering length $a_{B}$ to a small positive value or keeping the condensate density $n_{0}$ small. In Fig. 5(b) we also show the ratio of $V_{i, j}^{\alpha \beta}$ between on-site and nearest neighbor terms versus $\xi$. These ratios increase with the BEC healing length, indicating a longer effective range of 
the interactions. The effects of these interactions, which are beyond this paper, might include new ordered polaron phases in our system with Hamiltonian Eq. (25). As shown in Fig. 5(c), if we consider that the transverse confinement is due to a very deep optical lattice instead of a single well, different $1 \mathrm{D}$ tubes could then interact due to the bath-induced long range (attractive) interactions. A more rich phase diagram is expected in such mixeddimensional system coupled to a bosonic bath [108-111].

\section{LINDBLAD EQUATION AND INTER-BAND DYNAMICS}

The incoherent part of the Hamiltonian $\tilde{H}_{\text {inc }}=\tilde{H}-$ $\langle\tilde{H}\rangle_{T}$ includes the residual coupling between polarons and phonon bath:

$$
\begin{aligned}
\tilde{H}_{\mathrm{inc}} & =-\sum_{\langle i, j\rangle} \sum_{\alpha}\left(J^{\alpha} \hat{T}_{i, j}^{\alpha \alpha}\right) \hat{a}_{i}^{\alpha \dagger} \hat{a}_{j}^{\alpha}+\sum_{i} \sum_{\alpha}\left[\sum_{\mathbf{q}} \hbar \omega_{\mathbf{q}} \hat{b}_{\mathbf{q}}^{\dagger}\left(M_{i, \mathbf{q}}^{\alpha \alpha}-\Lambda_{i, \mathbf{q}}^{\alpha \alpha}\right)^{*}+h . c .\right] \hat{a}_{i}^{\alpha \dagger} \hat{a}_{i}^{\alpha} \\
& +\sum_{i} \sum_{\alpha \neq \beta}\left\{\sum_{\mathbf{q}} \hbar \omega_{\mathbf{q}}\left[\hat{b}_{\mathbf{q}}^{\dagger}\left(M_{i, \mathbf{q}}^{\alpha \beta}\right)^{*}\left(\hat{K}_{i, i}^{\alpha \beta}\right)-\left(\Lambda_{i, \mathbf{q}}^{\alpha \alpha}\right)\left(M_{i, \mathbf{q}}^{\alpha \beta}\right)^{*}\left(\hat{T}_{i, i}^{\alpha \beta}\right)\right]+h . c .\right\} \hat{a}_{i}^{\alpha \dagger} \hat{a}_{i}^{\beta} \\
& -\sum_{i \neq j} \sum_{\alpha} \sum_{\alpha^{\prime} \neq \beta^{\prime}}\left\{\sum_{\mathbf{q}} \hbar \omega_{\mathbf{q}}\left[\left(\Lambda_{i, \mathbf{q}}^{\alpha \alpha}\right)\left(M_{j, \mathbf{q}}^{\alpha^{\prime} \beta^{\prime}}\right)^{*}\left(\hat{T}_{j, j}^{\alpha^{\prime} \beta^{\prime}}\right)\right]+h . c .\right\} \hat{a}_{i}^{\alpha \dagger} \hat{a}_{i}^{\alpha} \hat{a}_{j}^{\alpha^{\prime} \dagger} \hat{a}_{j}^{\beta^{\prime}} .
\end{aligned}
$$

with $\hat{T}_{i, j}^{\alpha \beta} \equiv \hat{K}_{i, j}^{\alpha \beta}-\left\langle\hat{K}_{i, j}^{\alpha \beta}\right\rangle_{T}$. There are three different coupling terms in Eq. (39): 1) an intra-band part due to hopping of the polaron to nearest neighbor sites and residual phonon dressing effects; 2) an inter-band part due to polaron dynamics between two bands; and 3) a mixed many-body term due to effective interactions between polarons.

The coherent part of the Hamiltonian Eq. (25), as calculated before, is a two-band Hubbard Hamiltonian. In order to focus on inter-band relaxation effects, we restrict our investigations to the case of a single polaron and ignore the interaction part $\hat{V}_{\mathrm{P}}$. The incoherent Hamiltonian part Eq. (39) can be treated by a Lindblad master equation. After applying the Born-Markov approximation, the Lindblad equation has the form:

$$
\frac{d \rho(t)}{d t}=-i\left[\langle\tilde{H}\rangle_{T}, \rho(t)\right]+\mathcal{L}_{I}[\rho(t)]
$$

with reduced density operator $\rho(t)$ of the polaron system. All decoherence effects in the system are described by the dissipator:

$$
\begin{aligned}
\mathcal{L}_{I}[\rho(t)]= & \sum_{\alpha \beta} \sum_{\alpha^{\prime} \beta^{\prime}} \sum_{i, j} \sum_{i^{\prime}, j^{\prime}} \gamma_{i j ; i^{\prime} j^{\prime}}^{\alpha \beta ; \beta^{\prime}} \\
& \times\left(C_{i^{\prime} j^{\prime}}^{\alpha^{\prime} \beta^{\prime}} \rho C_{i j}^{\alpha \beta \dagger}-\frac{1}{2}\left\{C_{i j}^{\alpha \beta \dagger} C_{i^{\prime} j^{\prime}}^{\alpha^{\prime} \beta^{\prime}}, \rho\right\}\right)
\end{aligned}
$$

with the quantum jump operators $C_{i j}^{\alpha \beta} \equiv\left(\hat{a}_{i}^{\alpha \dagger} \hat{a}_{j}^{\beta}\right)$. These jump operators describe the dynamics of polarons instead of bare impurities, since they include the creation and annihilation operators of the polaron. In the inter- action picture with $\hat{O}(t) \equiv e^{-i\langle\tilde{H}\rangle_{T} t / \hbar} \hat{O} e^{i\langle\tilde{H}\rangle_{T} t / \hbar}$, the decoherence rates are defined as:

$$
\gamma_{i j ; i^{\prime} j^{\prime}}^{\alpha \beta ; \alpha^{\prime} \beta^{\prime}} \equiv 2 \operatorname{Re} \int_{0}^{\infty} d \tau e^{i \omega \tau} g_{i j ; i^{\prime} j^{\prime}}^{\alpha \beta ; \alpha^{\prime} \beta^{\prime}}(\tau)
$$

and the correlation functions $g_{i j ; i^{\prime} j^{\prime}}^{\alpha \beta ; \alpha^{\prime} \beta^{\prime}}(\tau)$ are defined in Appendix B.

In this section, we focus our study on inter-band spontaneous relaxation of the polaron. Due to longitudinal trapping, the energy scales for intra- and inter-band dynamics are mismatched as $J^{0} ; J^{1} \ll \varepsilon_{\mathrm{P}}^{\Delta}$. This allows us to use the rotating wave approximation (RWA) for interband dynamics and decouple it from intra-band dynamics in Eq. (B4). We then use the short-hand notation $\gamma_{i, j}^{01} \equiv \gamma_{i i ; j j}^{01 ; 01}$ and $C_{i}^{01} \equiv\left(\hat{a}_{i}^{0 \dagger} \hat{a}_{i}^{1}\right)$ to describe the polaron relaxation processes. Due to the coupling with the bath, a polaron in the upper band can spontaneously relax to the lower band with rate $\gamma_{i, j}^{01}$ and emit a phonon. In our system, the corresponding phonon energy $\hbar \omega_{\mathbf{q}}$ for interband dynamics is much larger than the BEC temperature $k_{\mathrm{B}} T$. For inter-band dynamics, the phonon bath temperature is thus effectively zero. In the interaction picture, this process is described by the master equation

$$
\frac{d}{d t} \hat{\rho} \equiv \sum_{i, j} \gamma_{i, j}^{01}\left(C_{j}^{01} \hat{\rho} C_{i}^{01 \dagger}-\frac{1}{2}\left\{C_{i}^{01 \dagger} C_{j}^{01}, \hat{\rho}\right\}\right)
$$

The single polaron spontaneous relaxation rate $\gamma_{i, j}^{01}$ is calculated in Eq. (B14). It is convenient to write the master equation for relaxation processes in momentum space, as 
long as $\gamma_{i, j}^{01}$ only depends on the value of $(i-j)$ :

$$
\frac{d}{d t} \hat{\rho} \equiv \sum_{q} \gamma_{q}\left(C_{q}^{01} \hat{\rho} C_{q}^{01 \dagger}-\frac{1}{2}\left\{C_{q}^{01 \dagger} C_{q}^{01}, \hat{\rho}\right\}\right)
$$

with $C_{q}^{01} \equiv \sum_{k}\left(\hat{a}_{k-q}^{0 \dagger} \hat{a}_{k}^{1}\right)$ and $\hat{a}_{k}^{\alpha}$ is the polaron annihilation operator in momentum space [112, 113]. Here, $k, k-q$ are the quasi-momenta in the first Brillouin zone and $C_{q} \equiv C_{q+z G}, z \in \mathbb{Z}, G=2 \pi / d$. The relaxation rate is written as a function of longitudinal phonon momentum:

$$
\gamma_{q}=\frac{1}{N} \sum_{(i-j)} \gamma_{i, j}^{01} e^{-i q(i-j) d}
$$

where $N$ is lattice number. When $q d \gg 2 \pi$, the sum over with $i \neq j$, involving polaron relaxation effects over different sites, decays rapidly with $|i-j|$.

The total relaxation rate $\gamma$ is a sum over longitudinal phonon momenta, $\gamma \equiv \sum_{q} \gamma_{q}$ [113]. From the expression of the correlation function $g_{i j}^{01}(\tau)$ in Eq. (B12), the polaronic inter-band relaxation contains two terms. The first term in (B12) is a single-phonon process, with the polaron absorbing (emitting) one phonon from (to) the bath. This term is similar to Fermi's Golden Rule except a renormalization factor $\left\langle\hat{K}_{i, i}^{10}(\tau) \hat{K}_{j, j}^{01}(0)\right\rangle_{T}$. The second term describes higher order processes involving two phonons begin absorbed (emitted), which are absent in Fermi's Golden Rule. In the total relaxation rate, we will only consider the leading-order single-phonon process in Eq. (B14) and neglect the higher-order processes. At zero temperature, only spontaneous emission of phonons is allowed with the single polaron spontaneous relaxation rate

$$
\begin{aligned}
\gamma^{\mathrm{P}}=2 \operatorname{Re} & \int_{0}^{\infty} d \tau e^{i \varepsilon_{\mathrm{P}}^{\Delta} \tau / \hbar} \sum_{\mathbf{q}} \omega_{\mathbf{q}}^{2}\left|M_{\mathbf{q}}^{01}\right|^{2} e^{-i \omega_{\mathbf{q}} \tau} \\
& \times\left\langle\hat{K}_{i, i}^{10}(\tau) \hat{K}_{i, i}^{01}(0)\right\rangle_{T}
\end{aligned}
$$

where $\left\langle\hat{K}_{i, i}^{10}(\tau) \hat{K}_{i, i}^{01}(0)\right\rangle_{T}$ is defined in Eq. (B13) as a renormalization factor for inter-band relaxation dynamics.

In order to investigate how polaron effects affect the inter-band dynamics, we also derive a Lindblad equation for the original "bare" Hamiltonian in Eq. (19), before the Lang-Firsov transformation had been applied. The corresponding single-impurity spontaneous relaxation rate in this case is

$$
\gamma^{0}=2 \operatorname{Re} \int_{0}^{\infty} d \tau e^{i \varepsilon^{\Delta} \tau / \hbar} \sum_{\mathbf{q}} \omega_{\mathbf{q}}^{2}\left|M_{\mathbf{q}}^{01}\right|^{2} e^{-i \omega_{\mathbf{q}} \tau}
$$

and reduces to Fermi's Golden Rule formula:

$$
\gamma^{0}=2 \pi \sum_{\mathbf{q}} \omega_{\mathbf{q}}^{2}\left|M_{\mathbf{q}}^{01}\right|^{2} \delta\left(\hbar \omega_{\mathbf{q}}-\varepsilon^{\Delta}\right) .
$$

Comparing Eq. (44) and Eq. (45), we observe that the renormalization of the band gap from $\varepsilon^{\Delta}$ to $\varepsilon_{\mathrm{P}}^{\Delta}$ increases the energy of the phonon which is created, due to energy conservation. The polaron relaxation rate $\gamma^{\mathrm{P}}$ is also reduced by an additional exponential factor, since the coupling between impurity and bath is also renormalized.

Before we discuss the numerical results for the relaxation rate, let us first look at the behavior of $\gamma_{q}$ as a function of phonon momentum in the longitudinal direction. For the bare particle relaxation rate, the prediction of Fermi's golden rule gives:

$$
\begin{aligned}
\gamma_{q}^{0} & \equiv 2 \operatorname{Re} \int_{0}^{\infty} d \tau e^{i \varepsilon^{\Delta} \tau / \hbar} \sum_{q_{y}, q_{z}} \omega_{\mathbf{q}}^{2}\left|M_{\mathbf{q}}^{01}\right|^{2} e^{-i \omega_{\mathbf{q}} \tau} \\
& \approx 2 \pi \sum_{q_{\perp}} \omega_{\mathbf{q}}^{2}\left|M_{\mathbf{q}}^{01}\right|^{2} \delta\left(\hbar \omega_{\mathbf{q}}-\varepsilon^{\Delta}\right)
\end{aligned}
$$

In a deep lattice, the impurity-phonon coupling matrices $M_{\mathbf{q}}^{\alpha \beta}$ can be approximated by Gaussian functions in Eq. (15). Then the value of Fermi's golden rule for $\gamma_{q}^{0}$ in Eq. (47) is found to be:

$$
\begin{aligned}
\gamma_{q}^{0} \approx & \left(\frac{m_{B}}{\hbar^{2}}\right) \frac{n_{0}}{L_{x}} g_{I B}^{2} \\
& \times\left(q^{2} \sigma_{x}^{2} / 2\right) e^{-\left(q^{2} \sigma_{x}^{2}+q_{\perp}^{2} \sigma_{\perp}^{2}\right) / 2}
\end{aligned}
$$

where $L_{x}$ is the phonon quantization length (analogous to the quantization volume $\Omega$ ) in the longitudinal direction. The phonon momentum in the transverse direction $q_{\perp}$ is fixed by energy conservation $\hbar \omega_{\mathbf{q}}=\varepsilon^{\Delta} \approx \hbar \omega_{x}$, where $|\mathbf{q}|^{2}=q^{2}+q_{\perp}^{2}$ and $\omega_{x}$ is the longitudinal oscillation frequency. An additional consequence of energy conservation, is that $\gamma_{q}$ is cut off when $\hbar \omega_{\mathbf{q}}(|\mathbf{q}|=q)=\hbar \omega_{x}$. The cut-off is

$$
\left|q \sigma_{x}\right| \approx \sqrt{2 \frac{m_{B}}{m_{I}}}
$$

which only depends on the mass ratio $m_{I} / m_{B}$ between impurity and BEC particles.

On the other hand, the polaron relaxation rate $\gamma_{q}^{\mathrm{P}}$ versus longitudinal phonon momentum needs to be calculated numerically from Eq. (43) and Eq. (B14). As we discussed before for the total relaxation rate Eq. (44), we consider only the single-phonon emitting processes and calculate $\gamma_{q}^{\mathrm{P}}$ as:

$$
\begin{aligned}
\gamma_{q}^{\mathrm{P}} \approx 2 \operatorname{Re} & \int_{0}^{\infty} d \tau e^{i \varepsilon_{\mathrm{P}}^{\Delta} \tau / \hbar} \sum_{q_{y}, q_{z}} \omega_{\mathbf{q}}^{2}\left|M_{\mathbf{q}}^{01}\right|^{2} e^{-i \omega_{\mathbf{q}} \tau} \\
\cdot\left\langle\hat{K}_{i, i}^{10}(\tau) \hat{K}_{i, i}^{01}(0)\right\rangle_{T} &
\end{aligned}
$$

Comparing with Eq. (47), the polaron relaxation rate has a similar behavior except for an additional renormalization factor. Due to energy conservation, $\gamma_{q}^{\mathrm{P}}$ also has a cut-off when $\hbar \omega_{\mathbf{q}}(|\mathbf{q}|=q)=\varepsilon_{\mathrm{P}}^{\Delta}$. The renormalized polaron band gap $\varepsilon_{\mathrm{P}}^{\Delta}$, which is larger than the bare gap $\varepsilon^{\Delta}$, will shift this cut-off position to higher phonon momentum. At the same time, the renormalization factor in Eq. (50) will reduce the value of $\gamma_{q}^{\mathrm{P}}$. 
In Fig. 6 we show the behavior of the relaxation rate $\gamma_{q}^{\mathrm{P}}$ and $\gamma_{q}^{0}$ as functions of longitudinal momentum $q \sigma_{x}$ with different impurity-BEC mass ratio. In order to compare the differences between $\gamma_{q}^{\mathrm{P}}$ and $\gamma_{q}^{0}$ at different coupling, we divide both $\gamma_{q}^{\mathrm{P}}$ and $\gamma_{q}^{0}$ by $\kappa^{2}$ and then normalize them. In this way, the Fermi's golden rule value $\gamma_{q}^{0}$ always stays the same for different coupling constants $\kappa$. As shown in Fig. 6, the dashed black lines indicate normalized $\gamma_{q}^{0}$ from Eq. (48), while the cut-off of $\left|q \sigma_{x}\right|$ in Eq. (49) is indicated by black lines. The total relaxation rate $\gamma^{0}=\sum_{q} \gamma_{q}^{0}$ from Fermi's golden rule is the gray area below the black curve. At $\kappa=0$, the polaronic relaxation rate $\gamma_{q}^{\mathrm{P}}$ is identical to the results from the Golden Rule.

On the other hand, as the impurity-BEC coupling is increased, the polaron relaxation rate $\gamma_{q}^{\mathrm{P}}$ from Eq. (50) is renormalized by the polaron band gap $\varepsilon_{\mathrm{P}}^{\Delta}$ and renormalization factor. The increased polaron band gap will involve more phonons by shifting the cut-off momentum (49), while the renormalization factor reduces the whole momentum range. In Fig. 6 we also show the polaron relaxation rate $\gamma_{q}^{\mathrm{P}}$ with blue (red) curve at $\kappa=1(2)$ and total relaxation rate $\gamma^{\mathrm{P}}$ with blue (red) area.

As shown in Fig. 6, the renormalization of the relaxation rate is different for various impurity-BEC mass ratios. For larger mass ratio (heavy impurity) such as a system with single ${ }^{133} \mathrm{Cs}$ impurity coupled with ${ }^{87} \mathrm{Rb}$ BEC, the polaron relaxation processes will be enhanced by the shift of the cut-off momentum and increase the total rate. On the other hand, for smaller mass ratio (light impurity) such as a ${ }^{6} \mathrm{Li}$ impurity coupled with ${ }^{23} \mathrm{Na} \mathrm{BEC}$, the higher momentum cut-off for $\left|q \sigma_{x}\right|$ is not so important due to Gaussian decay of $\gamma_{q}$. In this case, the renormalization factor will reduce $\gamma_{q}^{\mathrm{P}}$ as well as the total relaxation rate $\gamma^{\mathrm{P}}$. We can also expect that, for extremely strong impurity-BEC coupling, the total relaxation rate will be reduced due to this renormalization factor with any mass ratio.

Finally we compare the polaronic inter-band relaxation rate $\gamma^{\mathrm{P}}$ and Fermi Golden Rule results $\gamma^{0}$ in Fig. 7 as a function of coupling constant and for different impurityBEC mass ratios. As we have already seen in Fig. 6, the polaron formation will renormalize the inter-band relaxation rate differently, depending on the mass ratio.

We first consider the weak impurity-BEC coupling regime with small $\kappa$ in Fig. 7(a). For a heavy impurity coupled to a light BEC bath, the inter-band relaxation process involves more phonon modes and will be enhanced. The difference in the ratio $\gamma^{\mathrm{P}} / \gamma^{0}$ between polaronic relaxation and the Fermi Golden Rule result increases with coupling constant $\kappa$. On the other hand, for a light impurity coupled to a heavy BEC, the impurity is dressed by heavy phonons in each band and tends to localize in the same band. Although the inter-band relaxation process does involve more phonon modes than the Fermi Golden Rule result, this effect is highly suppressed due to Gaussian decay of $\gamma_{q}$ with high momentum. The ratio $\gamma^{\mathrm{P}} / \gamma^{0}$ will be reduced in this system. We also notice
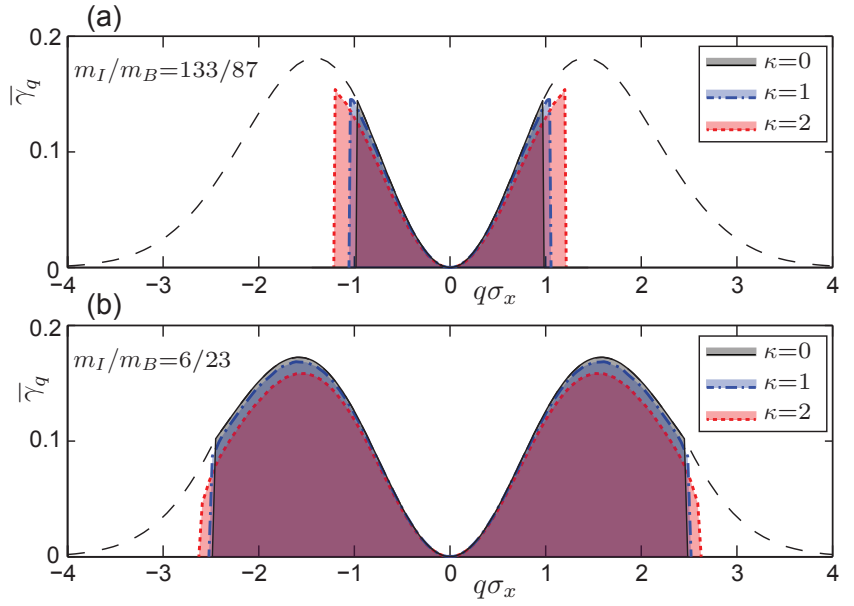

Figure 6: Normalized relaxation rate $\gamma_{q}^{\mathrm{P}}$ and $\gamma_{q}^{0}$ as functions of longitudinal momentum $q \sigma_{x}$ with different impurityBEC mass ratio. The dashed black lines indicate Fermi's Golden Rule results $\gamma_{q}^{0}$ from Eq. (48) with momentum cutoff given by the solid black line $(\kappa=0)$. The blue (red) curves indicate normalized polaron relaxation rate $\gamma_{q}^{\mathrm{P}}$ at coupling $\kappa=1(2)$. The total relaxation rates $\gamma=\sum_{q} \gamma_{q}$ are shown as the area below the corresponding curves. (a) System with $m_{I} / m_{B}=133 / 87$, i.e. ${ }^{133}$ Cs impurity in ${ }^{87} \mathrm{Rb}$ BEC. (b) System with $m_{I} / m_{B}=6 / 23$, i.e. ${ }^{6} \mathrm{Li}$ impurity in ${ }^{23} \mathrm{Na}$ BEC. Other parameters are $V_{I}^{x}=9 E_{R}, V_{I}^{\perp}=25 V_{I}^{x}$ and $a_{B}=100 a_{0}, n_{0}=1 \times 10^{14} \mathrm{~cm}^{-3}$.
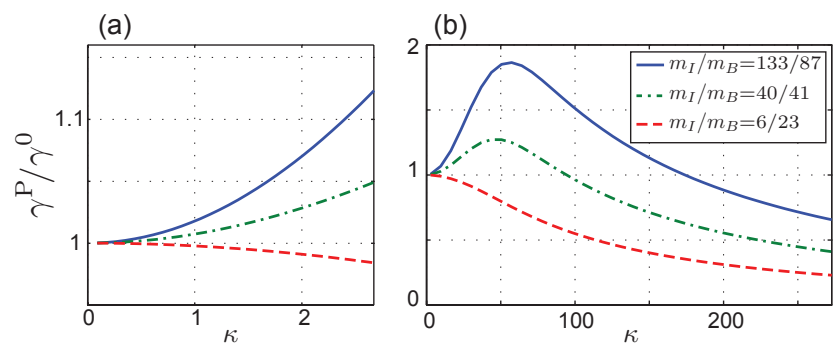

Figure 7: Ratio between polaron inter-band relaxation rate $\gamma^{\mathrm{P}}$ and Fermi Golden Rule results $\gamma^{0}$ at different impurityBEC mass ratio. (a) In the weak coupling regime, this ratio is increased with the coupling constant $\kappa$ for a heavy impurity coupled to a light BEC bath, and decreased for a light impurity coupled to a heavy BEC bath. The boson-boson scattering length here is chosen as $a_{B}=100 a_{0}$ and $\kappa_{c}=2.4$. (b) In the strong coupling regime, the polaron inter-band relaxation processes for any impurity-BEC mass ratio are suppressed. This leads to an inter-band self-trapping effect. The bosonboson scattering length here is $a_{B}=0.2 a_{0}$ and $\kappa_{c}=255$. We use $m_{I}=133, m_{B}=87$ for a ${ }^{133} \mathrm{Cs}$ impurity and ${ }^{87} \mathrm{Rb}$ BEC (blue line), $m_{I}=40, m_{B}=41$ for a ${ }^{40} \mathrm{~K}$ impurity and ${ }^{41} \mathrm{~K}$ BEC (green line), and $m_{I}=6, m_{B}=23$ for a ${ }^{6} \mathrm{Li}$ impurity and ${ }^{23} \mathrm{Na} \mathrm{BEC}$ (red line). Other parameters are $V_{I}^{x}=9 E_{R}, V_{I}^{\perp}=25 V_{I}^{x}$ and $n_{0}=1 \times 10^{14} \mathrm{~cm}^{-3}$. 
that the ratio $\gamma^{\mathrm{P}} / \gamma^{0}$ is not sensitive to the longitudinal trapping potential.

On the other hand, we can access the strong coupling regime by tuning the Bose-Bose scattering $a_{B}$ to a small positive value, since the allowed maximum coupling constant in Eq. (18) goes as $\kappa_{c} \propto 1 /\left(n_{0} a_{B}^{3}\right)^{1 / 4}$. For the strong impurity-BEC coupling region with large $\kappa$ in Fig. 7(b), though the Lindblad master equation might not be accurate enough for such strong inter-band coupling, we can still obtain some qualitative insight. In each band, the polaron is tightly dressed by phonons with different coupling strength, such that the polaron behaves as a quasi-particle with rather different properties in each band. Also, the band gap $\varepsilon_{\mathrm{P}}^{\Delta}$ is enhanced in comparison to the bare case, so the polaron cannot hop between bands by creating or annihilating phonons. As shown in Fig. 7(b), we indeed obtain a suppressed inter-band relaxation process. This inter-band self-trapping effect is expected in a strongly coupled impurity-BEC system. In a realistic impurity-BEC system, this effect might be also observed together with the well known self-trapping effect due to deformation of BEC [59, 88, 114-118].

\section{CONCLUSIONS}

In conclusion, we have studied a two-band Hamiltonian with Fröhlich impurity-phonon coupling with both intraand inter-band terms. Such a Hamiltonian can be realized in experiments where few impurities are immersed in a Bose-Einstein condensate of another species. The impurities are trapped by an anisotropic optical lattice behave as quasi-1D particles. Based on the Lang-Firsov transformation, we have derived and applied a variational two-band polaron transformation. We have calculated the coherent part of the resulting effective Hamiltonian with two (polaron) bands. In each band the impurity is dressed by phonons as a quasi-particle (polaron) with different properties. The polaronic intra-band coherent transport and polaron effective mass are both renormalized. Due to the coupling with bath, there are also induced on-site polaron energy shifts, and long-range interactions between different polarons.

In order to account for the residual incoherent coupling between polaron and bath, we have derived a Lindblad master equation, and focused on the single-polaron interband relaxation dynamics. Comparing to Fermi's Golden Rule calculations for the bare impurities, we found the renormalization of the relaxation rate to depend on the mass ratio of impurity and BEC particles. These polaronic effects in the inter-band relaxation dynamics might be observed in ongoing experiments. In the strong coupling limit of the two-band Fröhlich Hamiltonian, the impurity is inter-band self-trapped and can not tunnel between bands by creating or annihilating phonons.

\section{Acknowledgments}

The authors thank A. Daley, E. Demler, R. Gerritsma, F. Grusdt, G. Pupillo, T. Rentrop, R. Schmidt, T. Shi and A. Widera for fruitful discussions. Support by the Deutsche Forschungsgemeinschaft (DFG) via Sonderforschungsbereich SFB/TR 49, Forschergruppe FOR 801 and the high-performance computing center LOEWECSC is gratefully acknowledged.

\section{Appendix A: Two-band transformation and coherent Hamiltonian}

\section{Two-band polaron transformation}

In this section, we derive the two-band polaron transformation in detail. The transformation operator $\hat{S}$ in Eq. (20) can be written as $\hat{S}=\sum_{i, \alpha, \beta} \hat{C}_{i}^{\alpha, \beta} \hat{a}_{i}^{\alpha \dagger} \hat{a}_{i}^{\beta}$ with $\hat{C}_{i}^{\alpha, \beta} \equiv \sum_{\mathbf{q}} \Lambda_{\mathbf{q}}^{\alpha, \beta} e^{i \mathbf{q} \cdot \mathbf{R}_{i}}\left(\hat{b}_{-\mathbf{q}}^{\dagger}-\hat{b}_{\mathbf{q}}\right)$ or in matrix form as $\hat{\mathbf{C}}_{i} \equiv \sum_{\mathbf{q}} \boldsymbol{\Lambda}_{\mathbf{q}}\left(\hat{\mathbf{b}}_{-\mathbf{q}}^{\dagger}-\hat{\mathbf{b}}_{\mathbf{q}}\right) e^{i \mathbf{q} \cdot \mathbf{R}_{i}}$. By using the relation $\left[\hat{a}_{i}^{\alpha \dagger} \hat{a}_{i}^{\beta}, \hat{a}_{j}^{\gamma}\right]=-\delta_{i j} \delta_{\alpha \gamma} \hat{a}_{i}^{\beta}$, which is valid for both bosons and fermions, we obtain the transformed impurity annihilation operator as:

$$
\begin{aligned}
{\left[\hat{S}, \hat{a}_{i}^{\alpha}\right] } & =-\sum_{\beta}\left(\hat{\mathbf{C}}_{i}\right)_{\alpha \beta} \hat{a}_{i}^{\beta} ; \quad\left[\hat{S},\left[\hat{S}, \hat{a}_{i}^{\alpha}\right]\right]=+\sum_{\beta}\left(\hat{\mathbf{C}}_{i} \hat{\mathbf{C}}_{i}\right)_{\alpha \beta} \hat{a}_{i}^{\beta} ; \cdots ; \\
e^{\hat{S}} \hat{a}_{i}^{\alpha} e^{-\hat{S}} & =\sum_{\beta}\left(e^{-\hat{\mathbf{C}}_{i}}\right)_{\alpha \beta} \hat{a}_{i}^{\beta}=\sum_{\beta}\left(\hat{\mathbf{X}}_{i}\right)_{\alpha \beta} \hat{a}_{i}^{\beta},
\end{aligned}
$$


with $\hat{\mathbf{X}}_{i}=e^{-\hat{\mathbf{C}}_{i}}$ is defined in Eq. (21). The phonon annihilation operators can also be transformed in a similar way as:

$$
\begin{aligned}
{\left[\hat{S}, \hat{b}_{\mathbf{q}}\right] } & =\sum_{i, \alpha, \beta}\left(\left[\hat{\mathbf{C}}_{i}, \hat{\mathbf{b}}_{\mathbf{q}}\right]\right)_{\alpha, \beta} \hat{a}_{i}^{\alpha \dagger} \hat{a}_{i}^{\beta} ; \\
{\left[\hat{S},\left[\hat{S}, \hat{b}_{\mathbf{q}}\right]\right] } & =\left[\sum_{i, \alpha, \beta} \hat{C}_{i}^{\alpha, \beta} \hat{a}_{i}^{\alpha \dagger} \hat{a}_{i}^{\beta}, \sum_{i^{\prime}, \alpha^{\prime}, \beta^{\prime}}\left(\left[\hat{\mathbf{C}}_{i^{\prime}}, \hat{\mathbf{b}}_{\mathbf{q}}\right]\right)_{\alpha^{\prime}, \beta^{\prime}} \hat{a}_{i^{\prime}}^{\alpha^{\prime} \dagger} \hat{a}_{i^{\prime}}^{\beta^{\prime}}\right]=\sum_{i, \alpha, \beta}\left(\left[\hat{\mathbf{C}}_{i},\left[\hat{\mathbf{C}}_{i}, \hat{\mathbf{b}}_{\mathbf{q}}\right]\right]\right)_{\alpha \beta} \hat{a}_{i}^{\alpha \dagger} \hat{a}_{i}^{\beta} ;
\end{aligned}
$$

and thus

$$
e^{\hat{S}} \hat{b}_{\mathbf{q}} e^{-\hat{S}}=\hat{b}_{\mathbf{q}}+\sum_{i, \alpha, \beta}\left(e^{\hat{\mathbf{C}}_{i}} \hat{\mathbf{b}}_{\mathbf{q}} e^{-\hat{\mathbf{C}}_{i}}-\hat{\mathbf{b}}_{\mathbf{q}}\right)_{\alpha \beta} \hat{a}_{i}^{\alpha \dagger} \hat{a}_{i}^{\beta}=\hat{b}_{\mathbf{q}}+\sum_{i, \alpha, \beta}\left(\hat{\mathbf{X}}_{i}^{\dagger} \hat{\mathbf{b}}_{\mathbf{q}} \hat{\mathbf{X}}_{i}-\hat{b}_{\mathbf{q}}\right)_{\alpha \beta} \hat{a}_{i}^{\alpha \dagger} \hat{a}_{i}^{\beta}
$$

Different from the single band case, all higher commutators in Eq. (A5) are nonzero except when $\left[\boldsymbol{\Lambda}_{\mathbf{q}}, \boldsymbol{\Lambda}_{\mathbf{q}^{\prime}}\right]=0$. The Hamiltonian is transformed as:

$$
\begin{aligned}
\tilde{H} & =-\sum_{\langle i, j\rangle} \sum_{\alpha \beta}\left(\hat{\mathbf{X}}_{i}^{\dagger} \mathbf{J} \hat{\mathbf{X}}_{j}\right)_{\alpha \beta} \hat{a}_{i}^{\alpha \dagger} \hat{a}_{j}^{\beta}+\sum_{i} \sum_{\alpha \beta}\left(\hat{\mathbf{X}}_{i}^{\dagger} \varepsilon \hat{\mathbf{X}}_{i}\right)_{\alpha \beta} \hat{a}_{i}^{\alpha \dagger} \hat{a}_{i}^{\beta} \\
& +\sum_{\mathbf{q}} \hbar \omega_{\mathbf{q}}\left(\hat{b}_{\mathbf{q}}^{\dagger}+\sum_{i, \alpha, \beta}\left(\hat{\mathbf{X}}_{i}^{\dagger} \hat{\mathbf{b}}_{\mathbf{q}}^{\dagger} \hat{\mathbf{X}}_{i}-\hat{\mathbf{b}}_{\mathbf{q}}^{\dagger}\right)_{\alpha \beta} \hat{a}_{i}^{\alpha \dagger} \hat{a}_{i}^{\beta}\right)\left(\hat{b}_{\mathbf{q}}+\sum_{j, \alpha^{\prime}, \beta^{\prime}}\left(\hat{\mathbf{X}}_{j}^{\dagger} \hat{\mathbf{b}}_{\mathbf{q}} \hat{\mathbf{X}}_{j}-\hat{\mathbf{b}}_{\mathbf{q}}\right)_{\alpha^{\prime} \beta^{\prime}} \hat{a}_{j}^{\alpha^{\prime \dagger}} \hat{a}_{j}^{\beta^{\prime}}\right) \\
& +\sum_{i, \alpha, \beta} \sum_{\mathbf{q}} \hbar \omega_{\mathbf{q}}\left(\hat{b}_{-\mathbf{q}}^{\dagger}+\sum_{j, \alpha^{\prime}, \beta^{\prime}}\left(\hat{\mathbf{X}}_{j}^{\dagger} \hat{\mathbf{b}}_{-\mathbf{q}}^{\dagger} \hat{\mathbf{X}}_{j}-\hat{\mathbf{b}}_{-\mathbf{q}}^{\dagger}\right)_{\alpha^{\prime} \beta^{\prime}} \hat{a}_{j}^{\alpha^{\prime} \dagger} \hat{a}_{j}^{\beta^{\prime}}+\hat{b}_{\mathbf{q}}+\sum_{j, \alpha^{\prime}, \beta^{\prime}}\left(\hat{\mathbf{X}}_{j}^{\dagger} \hat{\mathbf{b}}_{\mathbf{q}} \hat{\mathbf{X}}_{j}-\hat{\mathbf{b}}_{\mathbf{q}}\right)_{\alpha^{\prime} \beta^{\prime}} \hat{a}_{j}^{\alpha^{\prime} \dagger} \hat{a}_{j}^{\beta^{\prime}}\right) \\
& \cdot\left(\hat{\mathbf{X}}_{i}^{\dagger} \mathbf{M}_{i, \mathbf{q}} \hat{\mathbf{X}}_{i}\right)_{\alpha \beta} \hat{a}_{i}^{\alpha \dagger} \hat{a}_{i}^{\beta} .
\end{aligned}
$$

The resulting polaron Hamiltonian is presented in Eq. (24).

\section{Coherent Hamiltonian under thermal average}

For completeness, we describe a general calculation of coherent Hamiltonian after two-band transformation. We apply a shorthand notation technique, which was used by Sibley and Munn [102, 103], to our two-band system. The transformed Hamiltonian in Eq. (19) is complicated since it has no assumptions for the variational parameters $\boldsymbol{\Lambda}_{\mathbf{q}}$ except the symmetry relations in Eq. (14). On the other hand, for our specific choice of assuming diagonal matrices for the variational parameters, all of the expressions in this section can be shown to be straightforward and simple.

The coherent part $\langle\tilde{H}\rangle_{T}$ of Eq. (19) can be calculated by averaging over the phonon bath. Here we assume the phonon bath is thermally distributed at the BEC temperature. From Eq. (19) we find these calculations require several types of thermal average values such as (I) $\left\langle\left(\hat{\mathbf{X}}_{i}^{\dagger} \mathbf{J} \hat{\mathbf{X}}_{j}\right)\right\rangle_{T}$, (II) $\left\langle\left(\hat{\mathbf{X}}_{i}^{\dagger} \hat{\mathbf{b}}_{\mathbf{q}}^{\dagger} \mathbf{M}_{i, \mathbf{q}}^{\dagger} \hat{\mathbf{X}}_{i}\right)\right\rangle_{T}$, (III) $\left\langle\left(\hat{\mathbf{X}}_{i}^{\dagger} \hat{\mathbf{b}}_{\mathbf{q}}^{\dagger} \hat{\mathbf{b}}_{\mathbf{q}} \hat{\mathbf{X}}_{i}\right)\right\rangle_{T}$ and $(\mathrm{IV})\left\langle\left(\hat{\mathbf{X}}_{i}^{\dagger} \hat{\mathbf{b}}_{\mathbf{q}}^{\dagger} \hat{\mathbf{X}}_{i}-\hat{\mathbf{b}}_{\mathbf{q}}^{\dagger}\right)_{\alpha \beta}\left(\hat{\mathbf{X}}_{i}^{\dagger} \hat{\mathbf{b}}_{\mathbf{q}} \hat{\mathbf{X}}_{i}-\hat{\mathbf{b}}_{\mathbf{q}}\right)_{\alpha^{\prime} \beta^{\prime}}\right\rangle_{T}$. Note that some terms inside $\langle(\cdots)\rangle_{T}$ are $2 \times 2$ matrices. Here we will discuss these terms in details, without making any assumption for the parameters $\boldsymbol{\Lambda}_{\mathbf{q}}$ except that they obey the same symmetry relations as for $\mathbf{M}_{\mathbf{q}}$ as given in Eq. (14).

$$
\text { Part I: }\left\langle\left(\hat{\mathbf{X}}_{i}^{\dagger} \mathbf{J} \hat{\mathbf{X}}_{j}\right)\right\rangle_{T} \text { and }\left\langle\left(\hat{\mathbf{X}}_{i}^{\dagger} \varepsilon \hat{\mathbf{X}}_{j}\right)\right\rangle_{T}
$$

The first coherent part describes a matrix $\mathbf{J}$ or $\varepsilon$ transformed by operator $\hat{\mathbf{X}}_{i}$. We use a general $2 \times 2$ matrix $\mathcal{F}$ instead of $J$ or $\varepsilon$ as:

$$
\left\langle\left(\hat{\mathbf{X}}_{i}^{\dagger} \mathcal{F} \hat{\mathbf{X}}_{j}\right)_{\alpha \beta}\right\rangle_{T}=\sum_{\alpha^{\prime} \beta^{\prime}}(\mathcal{F})_{\alpha^{\prime} \beta^{\prime}}\left\langle\left(\hat{\mathbf{X}}_{i}^{\dagger}\right)_{\alpha \alpha^{\prime}}\left(\hat{\mathbf{X}}_{j}\right)_{\beta^{\prime} \beta}\right\rangle_{T} \equiv \sum_{\alpha^{\prime} \beta^{\prime}}(\mathcal{F})_{\alpha^{\prime} \beta^{\prime}}\left\langle\left(e^{\hat{\mathbf{C}}_{i}-\hat{\mathbf{C}}_{j}}\right)_{\alpha \alpha^{\prime} ; \beta^{\prime} \beta}\right\rangle_{T}
$$


In the last step, we apply the shorthand notation technique and imply index $\alpha, \alpha^{\prime}$ for all functions of $\hat{\mathbf{C}}_{i}$ and the index $\beta^{\prime}, \beta$ for all functions of $\hat{\mathbf{C}}_{j}[99,102]$. This shorthand notation ignores the commutation of matrices and collects all terms of $\hat{\mathbf{C}}_{i}$ together and all $\hat{\mathbf{C}}_{j}$ together, before applying the indices. For an arbitrary operator $\hat{\mathbf{C}}$ and an arbitrary type of averaging involved, the expansion up to the second-order cumulant reads

$$
\langle\exp \hat{\mathbf{C}}\rangle_{T} \simeq \exp \left\{\langle\hat{\mathbf{C}}\rangle_{T}+\frac{1}{2}\left(\left\langle\hat{\mathbf{C}}^{2}\right\rangle_{T}-\langle\hat{\mathbf{C}}\rangle_{T}^{2}\right)\right\}
$$

This expansion is exact if applied without truncation directly to equation (A7) when the matrices $\hat{\mathbf{C}}$ commute, i.e. $\left[\hat{\mathbf{C}}_{i}, \hat{\mathbf{C}}_{j}\right]=0$. However, we make the approximation [99, 102], that the exponential in the short-hand notation also follows such a cumulant expansion. For small deviations from community, this will be a negligible approximation. Since the operators $\hat{\mathbf{C}}_{i}=\sum_{\mathbf{q}} \boldsymbol{\Lambda}_{\mathbf{q}} e^{i \mathbf{q} \cdot \mathbf{R}_{i}}\left(\hat{\mathbf{b}}_{-\mathbf{q}}^{\dagger}-\hat{\mathbf{b}}_{\mathbf{q}}\right)$ contain only creation and annihilation operators, the coherent part $\langle\hat{\mathbf{C}}\rangle_{T}=0$. In this case, we have $\langle\exp \hat{\mathbf{C}}\rangle_{T} \simeq \exp \left(\left\langle\hat{\mathbf{C}}^{2}\right\rangle_{T} / 2\right)$.

For the single band calculation, this relation reduces to the Bloch identity and is valid exactly [119]. However, in the two band system this relation is only an approximation because higher order terms are also present. The accuracy depends on the commutator $\left[\boldsymbol{\Lambda}_{\mathbf{q}}, \boldsymbol{\Lambda}_{\mathbf{q}^{\prime}}\right]$. Here we first apply this approximation with general parameters $\boldsymbol{\Lambda}_{\mathbf{q}}$ :

$$
\begin{aligned}
& \left\langle\left(e^{\hat{\mathbf{C}}_{i}-\hat{\mathbf{C}}_{j}}\right)_{\alpha \alpha^{\prime} ; \beta^{\prime} \beta}\right\rangle_{T} \simeq\left(e^{\frac{1}{2}\left\langle\left(\hat{\mathbf{C}}_{i}-\hat{\mathbf{C}}_{j}\right)^{2}\right\rangle_{T}}\right)_{\alpha \alpha^{\prime} ; \beta^{\prime} \beta}=\left(e^{\frac{1}{2}\left\langle\hat{\mathbf{C}}_{i}^{2}\right\rangle_{T}+\frac{1}{2}\left\langle-\hat{\mathbf{C}}_{i} \hat{\mathbf{C}}_{j}-\hat{\mathbf{C}}_{j} \hat{\mathbf{C}}_{i}\right\rangle_{T}+\frac{1}{2}\left\langle\hat{\mathbf{C}}_{j}^{2}\right\rangle_{T}}\right)_{\alpha \alpha^{\prime} ; \beta^{\prime} \beta} \\
= & \sum_{\alpha^{\prime \prime}, \beta^{\prime \prime}}\left(e^{\frac{1}{2}\left\langle\hat{\mathbf{C}}_{i}^{2}\right\rangle_{T}}\right)_{\alpha \alpha^{\prime \prime}}\left(e^{\frac{1}{2}\left\langle-\hat{\mathbf{C}}_{i} \hat{\mathbf{C}}_{j}-\hat{\mathbf{C}}_{j} \hat{\mathbf{C}}_{i}\right\rangle_{T}}\right)_{\alpha^{\prime \prime} \alpha^{\prime} ; \beta^{\prime} \beta^{\prime \prime}}\left(e^{\frac{1}{2}\left\langle\hat{\mathbf{C}}_{j}^{2}\right\rangle_{T}}\right)_{\beta^{\prime \prime} \beta} .
\end{aligned}
$$

The exponents above contains matrices $\left\langle\hat{\mathbf{C}}_{i}^{2}\right\rangle_{T},\left\langle\hat{\mathbf{C}}_{j}^{2}\right\rangle_{T},\left\langle\hat{\mathbf{C}}_{i} \hat{\mathbf{C}}_{j}\right\rangle_{T}$, which do not commute with each other. Fortunately, by using the shorthand notation $(\cdots)_{\alpha \alpha^{\prime} ; \beta^{\prime} \beta}$, those matrix elements with index $\alpha \alpha^{\prime} ; \beta^{\prime} \beta$ do commute between each other. They can be separated into independent exponents. Thermal averages such as $\left\langle\hat{\mathbf{C}}_{i}^{2}\right\rangle_{T}$ can be calculated easily:

$$
\left\langle\hat{\mathbf{C}}_{i}^{2}\right\rangle_{T}=\left\langle\sum_{\mathbf{q}} \sum_{\mathbf{q}^{\prime}} \boldsymbol{\Lambda}_{i, \mathbf{q}}^{\dagger} \boldsymbol{\Lambda}_{i, \mathbf{q}^{\prime}} \hat{\mathbf{b}}_{\mathbf{q}}^{\dagger} \hat{\mathbf{b}}_{\mathbf{q}^{\prime}}+\sum_{\mathbf{q}} \sum_{\mathbf{q}^{\prime}} \boldsymbol{\Lambda}_{i, \mathbf{q}^{\prime}} \boldsymbol{\Lambda}_{i, \mathbf{q}}^{\dagger} \hat{\mathbf{b}}_{\mathbf{q}^{\prime}} \hat{\mathbf{b}}_{\mathbf{q}}^{\dagger}\right\rangle_{T}=\sum_{\mathbf{q}}\left(\boldsymbol{\Lambda}_{i, \mathbf{q}}^{\dagger} \boldsymbol{\Lambda}_{i, \mathbf{q}}\right)\left(2 N_{\mathbf{q}}+1\right)
$$

where $N_{\mathbf{q}} \equiv\left(\exp \left(\hbar \omega_{\mathbf{q}} / k_{\mathrm{B}} T\right)-1\right)^{-1}$ is the thermally averaged phonon occupation number. The result of $\left\langle\hat{\mathbf{C}}_{i}^{2}\right\rangle_{T}$ is guaranteed to be a real number because of the symmetry relations $\boldsymbol{\Lambda}_{i, \mathbf{q}}=\boldsymbol{\Lambda}_{i,-\mathbf{q}}^{\dagger}$. The thermal averages in Eq. (A9) can be calculated as:

$$
\left\langle\left(e^{\hat{\mathbf{C}}_{i}-\hat{\mathbf{C}}_{j}}\right)_{\alpha \alpha^{\prime} ; \beta^{\prime} \beta}\right\rangle_{T} \simeq \sum_{\alpha^{\prime \prime}, \beta^{\prime \prime}}\left(e^{-\sum_{\mathbf{q}}\left(N_{\mathbf{q}}+\frac{1}{2}\right) \cdot\left(\boldsymbol{\Lambda}_{i, \mathbf{q}}^{\dagger} \boldsymbol{\Lambda}_{i, \mathbf{q}}\right)}\right)_{\alpha \alpha^{\prime \prime}}\left(e^{\sum_{\mathbf{q}}\left(2 N_{\mathbf{q}}+1\right)\left(\boldsymbol{\Lambda}_{i, \mathbf{q}}^{\dagger} \boldsymbol{\Lambda}_{j, \mathbf{q}}\right)}\right)_{\alpha^{\prime \prime} \alpha^{\prime} ; \beta^{\prime} \beta^{\prime \prime}}\left(e^{-\sum_{\mathbf{q}}\left(N_{\mathbf{q}}+\frac{1}{2}\right) \cdot\left(\boldsymbol{\Lambda}_{j, \mathbf{q}}^{\dagger} \mathbf{\Lambda}_{j, \mathbf{q}}\right)}\right)_{\beta^{\prime \prime} \beta} .
$$

The first and third term are easy to calculate. Then we expand the middle exponential under the shorthand notation $(\cdots)_{\alpha^{\prime \prime} \alpha^{\prime} ; \beta^{\prime} \beta^{\prime \prime}}$ :

$$
\begin{aligned}
& \left(e^{\sum_{\mathbf{q}}\left(2 N_{\mathbf{q}}+1\right)\left(\boldsymbol{\Lambda}_{i, \mathbf{q}}^{\dagger} \boldsymbol{\Lambda}_{j, \mathbf{q}}\right)}\right)_{\alpha^{\prime \prime} \alpha^{\prime} ; \beta^{\prime} \beta^{\prime \prime}}=\delta_{\alpha^{\prime \prime} \alpha^{\prime}} \delta_{\beta^{\prime} \beta^{\prime \prime}}+\frac{1}{1 !} \sum_{\mathbf{q}}\left(2 N_{\mathbf{q}}+1\right)\left(\boldsymbol{\Lambda}_{i, \mathbf{q}}^{\dagger}\right)_{\alpha^{\prime \prime} \alpha^{\prime}}\left(\boldsymbol{\Lambda}_{j, \mathbf{q}}\right)_{\beta^{\prime} \beta^{\prime \prime}} \\
& +\frac{1}{2 !} \sum_{\mathbf{q} \mathbf{q}^{\prime}}\left(2 N_{\mathbf{q}}+1\right)\left(2 N_{\mathbf{q}^{\prime}}+1\right)\left(\boldsymbol{\Lambda}_{i, \mathbf{q}}^{\dagger} \boldsymbol{\Lambda}_{i, \mathbf{q}^{\prime}}^{\dagger}\right)_{\alpha^{\prime \prime} \alpha^{\prime}}\left(\boldsymbol{\Lambda}_{j, \mathbf{q}} \boldsymbol{\Lambda}_{j, \mathbf{q}^{\prime}}\right)_{\beta^{\prime} \beta^{\prime \prime}}+\cdots \\
& +\frac{1}{k !} \sum_{\mathbf{q q}^{\prime} \cdots \mathbf{q}^{(k-1)}} \underbrace{\left(2 N_{\mathbf{q}}+1\right) \cdots\left(2 N_{\mathbf{q}^{(k-1)}}+1\right)}_{k} \underbrace{\left(\boldsymbol{\Lambda}_{i, \mathbf{q}}^{\dagger} \boldsymbol{\Lambda}_{i, \mathbf{q}^{\prime}}^{\dagger} \cdots \boldsymbol{\Lambda}_{i, \mathbf{q}^{(k-1)}}^{\dagger}\right)_{\alpha^{\prime \prime} \alpha^{\prime}}}_{k} \underbrace{\left(\boldsymbol{\Lambda}_{j, \mathbf{q}} \boldsymbol{\Lambda}_{j, \mathbf{q}^{\prime}} \cdots \boldsymbol{\Lambda}_{j, \mathbf{q}^{(k-1)}}\right)_{\beta^{\prime} \beta^{\prime \prime}}}_{k} .
\end{aligned}
$$

In each term, we combine elements with the same momentum q: 


$$
\begin{aligned}
& \left(e^{\sum_{\mathbf{q}}\left(2 N_{\mathbf{q}}+1\right)\left(\boldsymbol{\Lambda}_{i, \mathbf{q}}^{\dagger} \boldsymbol{\Lambda}_{j, \mathbf{q}}\right)}\right)_{\alpha^{\prime \prime} \alpha^{\prime} ; \beta^{\prime} \beta^{\prime \prime}}=\delta_{\alpha^{\prime \prime} \alpha^{\prime}} \delta_{\beta^{\prime} \beta^{\prime \prime}}+\frac{1}{1 !} \sum_{\mathbf{q}}\left(2 N_{\mathbf{q}}+1\right)\left(\boldsymbol{\Lambda}_{i, \mathbf{q}}^{\dagger}\right)_{\alpha^{\prime \prime} \alpha^{\prime}}\left(\boldsymbol{\Lambda}_{j, \mathbf{q}}\right)_{\beta^{\prime} \beta^{\prime \prime}} \\
& +\frac{1}{2 !} \sum_{\alpha^{(3)}, \beta^{(3)}}\left(\sum_{\mathbf{q}}\left(2 N_{\mathbf{q}}+1\right)\left(\boldsymbol{\Lambda}_{i, \mathbf{q}}^{\dagger}\right)_{\alpha^{\prime \prime} \alpha^{(3)}}\left(\boldsymbol{\Lambda}_{j, \mathbf{q}}\right)_{\beta^{\prime} \beta^{(3)}}\right)\left(\sum_{\mathbf{q}^{\prime}}\left(2 N_{\mathbf{q}^{\prime}}+1\right)\left(\boldsymbol{\Lambda}_{i, \mathbf{q}^{\prime}}^{\dagger}\right)_{\alpha^{(3)} \alpha^{\prime}}\left(\boldsymbol{\Lambda}_{j, \mathbf{q}^{\prime}}\right)_{\beta^{(3)} \beta^{\prime \prime}}\right)+\cdots \\
& +\frac{1}{k !} \underbrace{\sum_{\alpha^{(3)}, \beta^{(3)}} \cdots \sum_{\alpha^{(k+1)}, \beta^{(k+1)}}}_{k-1} \\
& \cdot \underbrace{\left(\sum_{\mathbf{q}}\left(2 N_{\mathbf{q}}+1\right)\left(\boldsymbol{\Lambda}_{i, \mathbf{q}}^{\dagger}\right)_{\alpha^{\prime \prime} \alpha^{(3)}}\left(\boldsymbol{\Lambda}_{j, \mathbf{q}}\right)_{\beta^{\prime} \beta^{(3)}}\right) \cdots\left(\sum_{\mathbf{q}^{(k-1)}}\left(2 N_{\mathbf{q}^{(k-1)}}+1\right)\left(\boldsymbol{\Lambda}_{i, \mathbf{q}^{(\mathbf{k}-1)}}^{\dagger}\right)_{\alpha^{(k+1)} \alpha^{\prime}}\left(\boldsymbol{\Lambda}_{\left.j, \mathbf{q}^{(\mathbf{k}-1)}\right)}\right)_{\beta^{(k+1) \beta^{\prime \prime}}}\right)}_{k}
\end{aligned}
$$

In order to simplify last part in Eq. (A13), we define 4 th rank tensors $\mathbf{G}_{i j}$ with elements:

$$
\left(\mathbf{G}_{i j}\right)_{\alpha^{\prime \prime} \alpha^{\prime} \beta^{\prime} \beta^{\prime \prime}} \equiv \sum_{\mathbf{q}}\left(2 N_{\mathbf{q}}+1\right)\left(\boldsymbol{\Lambda}_{i, \mathbf{q}}^{\dagger}\right)_{\alpha^{\prime \prime} \alpha^{\prime}}\left(\boldsymbol{\Lambda}_{j, \mathbf{q}}\right)_{\beta^{\prime} \beta^{\prime \prime}}
$$

and

$$
\left(\mathbf{G}_{i j}^{2}\right)_{\alpha \alpha^{\prime} \beta \beta^{\prime}} \equiv \sum_{\alpha^{\prime \prime} \beta^{\prime \prime}}\left(\mathbf{G}_{i j}\right)_{\alpha \alpha^{\prime \prime} \beta \beta^{\prime \prime}}\left(\mathbf{G}_{i j}\right)_{\alpha^{\prime \prime} \alpha^{\prime} \beta^{\prime \prime} \beta^{\prime}}
$$

Then the eq. (A13) can be written as:

$$
\begin{aligned}
& \left(e^{\sum_{\mathbf{q}}\left(2 N_{\mathbf{q}}+1\right)\left(\boldsymbol{\Lambda}_{i, \mathbf{q}}^{\dagger} \mathbf{\Lambda}_{j, \mathbf{q}}\right)}\right)_{\alpha^{\prime \prime} \alpha^{\prime} ; \beta^{\prime} \beta^{\prime \prime}} \\
= & \delta_{\alpha^{\prime \prime} \alpha^{\prime}} \delta_{\beta^{\prime} \beta^{\prime \prime}}+\frac{1}{1 !}\left(\mathbf{G}_{i j}\right)_{\alpha^{\prime \prime} \alpha^{\prime} \beta^{\prime} \beta^{\prime \prime}}+\frac{1}{2 !}\left(\mathbf{G}_{i j}^{2}\right)_{\alpha^{\prime \prime} \alpha^{\prime} \beta^{\prime} \beta^{\prime \prime}}+\frac{1}{k !}\left(\mathbf{G}_{i j}^{k}\right)_{\alpha^{\prime \prime} \alpha^{\prime} \beta^{\prime} \beta^{\prime \prime}} \\
= & \left(e^{\mathbf{G}_{i j}}\right)_{\alpha^{\prime \prime} \alpha^{\prime} \beta^{\prime} \beta^{\prime \prime}},
\end{aligned}
$$

where $\left(e^{\mathbf{G}_{i j}}\right)$ are also 4 th rank tensors.

The tensors $\mathbf{G}_{i j}$ involve only numbers, and thus $\left(e^{\mathbf{G}_{i j}}\right)$ can be calculated exactly. Finally, the first thermal average term can be calculated as:

$$
\begin{aligned}
\left\langle\left(\hat{\mathbf{X}}_{i}^{\dagger} \mathcal{F} \hat{\mathbf{X}}_{j}\right)_{\alpha \beta}\right\rangle_{T} & =\sum_{\alpha^{\prime} \beta^{\prime}}(\mathcal{F})_{\alpha^{\prime} \beta^{\prime}}\left\langle\left(e^{\hat{\mathbf{C}}_{i}-\hat{\mathbf{C}}_{j}}\right)_{\alpha \alpha^{\prime} ; \beta^{\prime} \beta}\right\rangle_{T} \\
\left\langle\left(e^{\hat{\mathbf{C}}_{i}-\hat{\mathbf{C}}_{j}}\right)_{\alpha \alpha^{\prime} ; \beta^{\prime} \beta}\right\rangle_{T} & =\sum_{\alpha^{\prime \prime}, \beta^{\prime \prime}}\left(e^{-\sum_{\mathbf{q}}\left(N_{\mathbf{q}}+\frac{1}{2}\right) \cdot\left(\boldsymbol{\Lambda}_{i, \mathbf{q}}^{\dagger} \boldsymbol{\Lambda}_{i, \mathbf{q}}\right)}\right)_{\alpha \alpha^{\prime \prime}}\left(e^{\mathbf{G}_{i j}}\right)_{\alpha^{\prime \prime} \alpha^{\prime} \beta^{\prime} \beta^{\prime \prime}}\left(e^{-\sum_{\mathbf{q}}\left(N_{\mathbf{q}}+\frac{1}{2}\right) \cdot\left(\boldsymbol{\Lambda}_{j, \mathbf{q}}^{\dagger} \mathbf{\Lambda}_{j, \mathbf{q}}\right)}\right)_{\beta^{\prime \prime} \beta} .
\end{aligned}
$$

$$
\text { Part II: }\left\langle\left(\hat{\mathbf{X}}_{i}^{\dagger} \hat{\mathbf{b}}_{\mathbf{q}}^{\dagger} \mathbf{M}_{i, \mathbf{q}}^{\dagger} \hat{\mathbf{X}}_{i}\right)\right\rangle_{T}
$$

Here we calculate the second type of thermal averages by the relation $f(x=1)=\int_{0}^{1} f^{\prime}(x) d x+f(x=0)$ :

$$
\begin{aligned}
& \left\langle\left(\hat{\mathbf{X}}_{i}^{\dagger} \hat{\mathbf{b}}_{\mathbf{q}}^{\dagger} \mathbf{M}_{i, \mathbf{q}}^{\dagger} \hat{\mathbf{X}}_{i}\right)\right\rangle_{T}=\left\langle\left(e^{\hat{\mathbf{C}}_{i}} \hat{\mathbf{b}}_{\mathbf{q}}^{\dagger} e^{-\hat{\mathbf{C}}_{i}}\right)\left(e^{\hat{\mathbf{C}}_{i}} \mathbf{M}_{i, \mathbf{q}}^{\dagger} e^{-\hat{\mathbf{C}}_{i}}\right)\right\rangle_{T} \\
& =\left\langle\int_{0}^{1} d x \frac{d}{d x}\left(e^{x \hat{\mathbf{C}}_{i}} \hat{\mathbf{b}}_{\mathbf{q}}^{\dagger} e^{-x \hat{\mathbf{C}}_{i}} e^{\hat{\mathbf{C}}_{i}} \mathbf{M}_{i, \mathbf{q}}^{\dagger} e^{-\hat{\mathbf{C}}_{i}}\right)\right\rangle_{T}+\left\langle\hat{\mathbf{b}}_{\mathbf{q}}^{\dagger} e^{\hat{\mathbf{C}}_{i}} \mathbf{M}_{i, \mathbf{q}}^{\dagger} e^{-\hat{\mathbf{C}}_{i}}\right\rangle_{T} \\
& =-\int_{0}^{1} d x\left\langle e^{x \hat{\mathbf{C}}_{i}} \boldsymbol{\Lambda}_{i, \mathbf{q}} e^{-x \hat{\mathbf{C}}_{i}} e^{\hat{\mathbf{C}}_{i}} \mathbf{M}_{i, \mathbf{q}}^{\dagger} e^{-\hat{\mathbf{C}}_{i}}\right\rangle_{T}+\left\langle\hat{\mathbf{b}}_{\mathbf{q}}^{\dagger} e^{\hat{\mathbf{C}}_{i}} \mathbf{M}_{i, \mathbf{q}}^{\dagger} e^{-\hat{\mathbf{C}}_{i}}\right\rangle_{T}
\end{aligned}
$$


where we have used the relation $\left[\hat{\mathbf{C}}_{i}, \hat{\mathbf{b}}_{\mathbf{q}}^{\dagger}\right]=-\boldsymbol{\Lambda}_{i, \mathbf{q}}$. The first term can be calculated by separating out the bath terms:

$$
\begin{aligned}
& \left\langle\left(e^{x \hat{\mathbf{C}}_{i}} \boldsymbol{\Lambda}_{i, \mathbf{q}} e^{-x \hat{\mathbf{C}}_{i}} e^{\hat{\mathbf{C}}_{i}} \mathbf{M}_{i, \mathbf{q}}^{\dagger} e^{-\hat{\mathbf{C}}_{i}}\right)_{\alpha \beta}\right\rangle_{T} \\
= & \sum_{\alpha^{\prime} \gamma \gamma^{\prime} \beta^{\prime}}\left\langle\left(e^{x \hat{\mathbf{C}}_{i}}\right)_{\alpha \alpha^{\prime}}\left(\boldsymbol{\Lambda}_{i, \mathbf{q}}\right)_{\alpha^{\prime} \gamma}\left(e^{(1-x) \hat{\mathbf{C}}_{i}}\right)_{\gamma \gamma^{\prime}}\left(\mathbf{M}_{i, \mathbf{q}}^{\dagger}\right)_{\gamma^{\prime} \beta^{\prime}}\left(e^{-\hat{\mathbf{C}}_{i}}\right)_{\beta^{\prime} \beta}\right\rangle_{T} \\
= & \sum_{\alpha^{\prime} \gamma \gamma^{\prime} \beta^{\prime}}\left(\boldsymbol{\Lambda}_{i, \mathbf{q}}\right)_{\alpha^{\prime} \gamma}\left(\mathbf{M}_{i, \mathbf{q}}^{\dagger}\right)_{\gamma^{\prime} \beta^{\prime}} \cdot\left\langle\left(e^{x \hat{\mathbf{C}}_{i}}\right)_{\alpha \alpha^{\prime}}\left(e^{(1-x) \hat{\mathbf{C}}_{i}}\right)_{\gamma \gamma^{\prime}}\left(e^{-\hat{\mathbf{C}}_{i}}\right)_{\beta^{\prime} \beta}\right\rangle_{T} .
\end{aligned}
$$

This element can be calculated by the same method as we applied for $\left\langle\left(e^{\hat{\mathbf{C}}_{i}}\right)_{\alpha \alpha^{\prime}}\left(e^{-\hat{\mathbf{C}}_{j}}\right)_{\beta^{\prime} \beta}\right\rangle_{T}$ in the previous section. Under the same shorthand notation:

$$
\begin{aligned}
& \left\langle\left(e^{x \hat{\mathbf{C}}_{i}}\right)_{\alpha \alpha^{\prime}}\left(e^{(1-x) \hat{\mathbf{C}}_{j}}\right)_{\gamma \gamma^{\prime}}\left(e^{-\hat{\mathbf{C}}_{k}}\right)_{\beta^{\prime} \beta}\right\rangle_{T}=\left\langle\left(e^{x \hat{\mathbf{C}}_{i}+(1-x) \hat{\mathbf{C}}_{j}-\hat{\mathbf{C}}_{k}}\right)_{\alpha \alpha^{\prime} ; \gamma \gamma^{\prime} ; \beta^{\prime} \beta}\right\rangle_{T} \\
\simeq & \left(e^{\frac{1}{2}\left\langle\left(x \hat{\mathbf{C}}_{i}+(1-x) \hat{\mathbf{C}}_{j}-\hat{\mathbf{C}}_{k}\right)^{2}\right\rangle_{T}}\right)_{\alpha \alpha^{\prime} ; \gamma \gamma^{\prime} ; \beta^{\prime} \beta} \\
= & \sum_{\alpha^{2} \alpha^{3} \gamma^{2} \gamma^{3}} \sum_{\beta^{2} \beta^{3}}\left(e^{\frac{1}{2}\left\langle x^{2} \hat{\mathbf{C}}_{i}^{2}\right\rangle_{T}}\right)_{\alpha \alpha^{(2)}}\left(e^{\frac{1}{2}\left\langle(1-x)^{2} \hat{\mathbf{C}}_{j}^{2}\right\rangle_{T}}\right)_{\gamma \gamma^{(2)}}\left(e^{\frac{1}{2}\left\langle\hat{\mathbf{C}}_{k}^{2}\right\rangle_{T}}\right)_{\beta^{\prime} \beta^{(2)}} \\
& \cdot\left(e^{\frac{1}{2}\left\langle x \hat{\mathbf{C}}_{i}(1-x) \hat{\mathbf{C}}_{j}\right\rangle_{T}}\right)_{\alpha^{(2)} \alpha^{(3)} ; \gamma^{(2)} \gamma^{(3)}}\left(e^{-\frac{1}{2}\left\langle x \hat{\mathbf{C}}_{i} \hat{\mathbf{C}}_{k}\right\rangle_{T}}\right)_{\alpha^{(3)} \alpha^{\prime} ; \beta(2) \beta^{(3)}}\left(e^{-\frac{1}{2}\left\langle(1-x) \hat{\mathbf{C}}_{j} \hat{\mathbf{C}}_{k}\right\rangle_{T}}\right)_{\gamma^{(3)} \gamma^{\prime} ; \beta^{(3)} \beta}
\end{aligned}
$$

with $\alpha, \beta, \gamma$ applied separately for $x \hat{\mathbf{C}}_{i},(1-x) \hat{\mathbf{C}}_{j}$ and $-\hat{\mathbf{C}}_{k}$. We have introduced the different indices $i, j$ and $k$ so that we can keep track of the terms required in applying the short-hand notation. In the end, we will set $i=j=k$ to recover the desired result. Using the results from previous section, these thermal averages will be:

$$
\begin{aligned}
& \left\langle\left(e^{x \hat{\mathbf{C}}_{i}}\right)_{\alpha \alpha^{\prime}}\left(e^{(1-x) \hat{\mathbf{C}}_{i}}\right)_{\gamma \gamma^{\prime}}\left(e^{-\hat{\mathbf{C}}_{i}}\right)_{\beta^{\prime} \beta}\right\rangle_{T} \\
= & \sum_{\alpha^{2} \alpha^{3} \gamma^{2} \gamma^{3} \beta^{2} \beta^{3}}\left(e^{-\sum_{\mathbf{q}}\left(N_{\mathbf{q}}+\frac{1}{2}\right) \cdot x^{2} \cdot \boldsymbol{\Lambda}_{\mathbf{q}}^{\dagger} \boldsymbol{\Lambda}_{\mathbf{q}}}\right)_{\alpha \alpha^{2}}\left(e^{-\sum_{\mathbf{q}}\left(N_{\mathbf{q}}+\frac{1}{2}\right) \cdot(1-x)^{2} \cdot \boldsymbol{\Lambda}_{\mathbf{q}}^{\dagger} \boldsymbol{\Lambda}_{\mathbf{q}}}\right)_{\gamma \gamma^{2}}\left(e^{-\sum_{\mathbf{q}}\left(N_{\mathbf{q}}+\frac{1}{2}\right) \cdot \boldsymbol{\Lambda}_{\mathbf{q}}^{\dagger} \boldsymbol{\Lambda}_{\mathbf{q}}}\right)_{\beta^{\prime} \beta^{2}} \\
& \cdot\left(e^{-x(1-x) \mathbf{G}_{i j}}\right)_{\alpha^{(2)} \alpha^{(3)} \gamma \gamma^{(3)}}\left(e^{x \mathbf{G}_{i k}}\right)_{\alpha^{(3)} \alpha^{\prime} \beta^{(2)} \beta^{(3)}}\left(e^{(1-x) \mathbf{G}_{j k}}\right)_{\gamma^{(3)} \gamma^{\prime} \beta^{(3)} \beta}
\end{aligned}
$$

with $\mathbf{G}_{i j}$ from Eq. (A14).

On the other hand, the second term $\left\langle\hat{\mathbf{b}}_{\mathbf{q}}^{\dagger} e^{\hat{\mathbf{C}}_{i}} \mathbf{M}_{i, \mathbf{q}}^{\dagger} e^{-\hat{\mathbf{C}}_{i}}\right\rangle_{T}$ in Eq. (A18) can be calculated as:

$$
\left\langle\left(\hat{\mathbf{b}}_{\mathbf{q}}^{\dagger} e^{\hat{\mathbf{C}}_{i}} \mathbf{M}_{i, \mathbf{q}}^{\dagger} e^{-\hat{\mathbf{C}}_{i}}\right)_{\alpha \beta}\right\rangle_{T}=\sum_{\alpha^{\prime} \beta^{\prime}}\left(\mathbf{M}_{i, \mathbf{q}}^{\dagger}\right)_{\alpha^{\prime} \beta^{\prime}}\left\langle\hat{b}_{\mathbf{q}}^{\dagger}\left(e^{\hat{\mathbf{C}}_{i}-\hat{\mathbf{C}}_{i}}\right)_{\alpha \alpha^{\prime} ; \beta^{\prime} \beta}\right\rangle_{T}
$$

From the results in [103], the elements such as $\left\langle\hat{\mathbf{b}}_{\mathbf{q}}^{\dagger}\left(e^{\hat{\mathbf{C}}_{i}-\hat{\mathbf{C}}_{i}}\right)\right\rangle_{T}$ can be obtained by differentiating $\left(e^{\hat{\mathbf{C}}_{i}-\hat{\mathbf{C}}_{i}}\right)$ before taking the thermal average. On the other hand, since the thermal average only involves the phonon degrees of freedom, we can switch the order and differentiate $\left\langle\left(e^{\hat{\mathbf{C}}_{i}-\hat{\mathbf{C}}_{i}}\right)\right\rangle_{T}$ after taking the thermal average. By comparing the two steps, we obtain the relation:

$$
\left\langle\hat{\mathbf{b}}_{\mathbf{q}}^{\dagger}\left(e^{\hat{\mathbf{C}}_{i}-\hat{\mathbf{C}}_{i}}\right)\right\rangle_{T}=-\left\langle\hat{\mathbf{b}}_{\mathbf{q}}^{\dagger} \sum_{\mathbf{q}^{\prime}} \boldsymbol{\Lambda}_{i, \mathbf{q}^{\prime}} \hat{\mathbf{b}}_{\mathbf{q}^{\prime}}\right\rangle_{T}\left\langle\left(e^{\hat{\mathbf{C}}_{i}-\hat{\mathbf{C}}_{i}}\right)\right\rangle_{T}+\left\langle\left(e^{\hat{\mathbf{C}}_{i}-\hat{\mathbf{C}}_{i}}\right)\right\rangle_{T}\left\langle\hat{\mathbf{b}}_{\mathbf{q}}^{\dagger} \sum_{\mathbf{q}^{\prime}} \boldsymbol{\Lambda}_{i, \mathbf{q}^{\prime}} \hat{\mathbf{b}}_{\mathbf{q}^{\prime}}\right\rangle_{T}
$$

which can be calculated by the results in Eq. (A17). As an example, we show a simplified calculation in Eq.(B11), where the transformation matrices $\boldsymbol{\Lambda}_{i, \mathbf{q}}$ are diagonal.

We finally obtain the second term in Eq. (A18):

$$
\begin{aligned}
& \left\langle\hat{\mathbf{b}}_{\mathbf{q}}^{\dagger} e^{\hat{\mathbf{C}}_{i}} \mathbf{M}_{i, \mathbf{q}}^{\dagger} e^{-\hat{\mathbf{C}}_{i}}\right\rangle_{T} \\
= & \sum_{\alpha^{\prime} \beta^{\prime}} N_{\mathbf{q}}\left(\mathbf{M}_{i, \mathbf{q}}^{\dagger}\right)_{\alpha^{\prime} \beta^{\prime}}\left[\sum_{\alpha^{\prime \prime}}\left(-\boldsymbol{\Lambda}_{i, \mathbf{q}}\right)_{\alpha \alpha^{\prime \prime}}\left\langle\left(e^{\hat{\mathbf{C}}_{i}-\hat{\mathbf{C}}_{i}}\right)_{\alpha^{\prime \prime} \alpha^{\prime} ; \beta^{\prime} \beta}\right\rangle_{T}+\sum_{\beta^{\prime \prime}}\left\langle\left(e^{\hat{\mathbf{C}}_{i}-\hat{\mathbf{C}}_{i}}\right)_{\alpha \alpha^{\prime} ; \beta^{\prime} \beta^{\prime \prime}}\right\rangle_{T}\left(\boldsymbol{\Lambda}_{i, \mathbf{q}}\right)_{\beta^{\prime \prime} \beta}\right] .
\end{aligned}
$$


Part III: $\left\langle\left(\hat{\mathbf{X}}_{i}^{\dagger} \hat{\mathbf{b}}_{\mathbf{q}}^{\dagger} \hat{\mathbf{b}}_{\mathbf{q}} \hat{\mathbf{X}}_{i}\right)\right\rangle_{T}$

This term can be calculated by a similar integral method as in the previous section:

$$
\begin{aligned}
& \left\langle\left(\hat{\mathbf{X}}_{i}^{\dagger} \hat{\mathbf{b}}_{\mathbf{q}}^{\dagger} \hat{\mathbf{b}}_{\mathbf{q}} \hat{\mathbf{X}}_{i}\right)\right\rangle_{T}=\int_{0}^{1} d y\left\langle\frac{d}{d y}\left(e^{y \hat{\mathbf{C}}_{i}} \hat{\mathbf{b}}_{\mathbf{q}}^{\dagger} \hat{\mathbf{b}}_{\mathbf{q}} e^{-y \hat{\mathbf{C}}_{i}}\right)\right\rangle_{T}+\left\langle\hat{\mathbf{b}}_{\mathbf{q}}^{\dagger} \hat{\mathbf{b}}_{\mathbf{q}}\right\rangle_{T} \\
= & -\int_{0}^{1} d y\left\langle e^{y \hat{\mathbf{C}}_{i}} \hat{\mathbf{b}}_{\mathbf{q}}^{\dagger} \boldsymbol{\Lambda}_{i, \mathbf{q}}^{\dagger} e^{-y \hat{\mathbf{C}}_{i}}+h . c .\right\rangle_{T}+\left\langle\hat{\mathbf{b}}_{\mathbf{q}}^{\dagger} \hat{\mathbf{b}}_{\mathbf{q}}\right\rangle_{T}
\end{aligned}
$$

Here the thermal average $\left\langle e^{y \hat{\mathbf{C}}_{i}} \hat{\mathbf{b}}_{\mathbf{q}}^{\dagger} \boldsymbol{\Lambda}_{i, \mathbf{q}}^{\dagger} e^{-y \hat{\mathbf{C}}_{i}}\right\rangle_{T}$ can be obtained by changing $\hat{\mathbf{C}}_{i}$ to $y \hat{\mathbf{C}}_{i}$ and $\mathbf{M}_{i, \mathbf{q}}$ to $\boldsymbol{\Lambda}_{i, \mathbf{q}}$ in Eq. (A18):

$$
\begin{aligned}
& \left\langle\left(e^{y \hat{\mathbf{C}}_{i}} \hat{\mathbf{b}}_{\mathbf{q}}^{\dagger} \boldsymbol{\Lambda}_{i, \mathbf{q}}^{\dagger} e^{-y \hat{\mathbf{C}}_{i}}\right)_{\alpha \beta}\right\rangle_{T} \\
= & -\int_{0}^{1} d x\left[\sum_{\alpha^{\prime} \gamma \gamma^{\prime} \beta^{\prime}} y\left(\boldsymbol{\Lambda}_{i, \mathbf{q}}\right)_{\alpha^{\prime} \gamma}\left(\boldsymbol{\Lambda}_{i, \mathbf{q}}^{\dagger}\right)_{\gamma^{\prime} \beta^{\prime}} \cdot\left\langle\left(e^{x y \hat{\mathbf{C}}_{i}}\right)_{\alpha \alpha^{\prime}}\left(e^{(1-x) y \hat{\mathbf{C}}_{i}}\right)_{\gamma \gamma^{\prime}}\left(e^{-y \hat{\mathbf{C}}_{i}}\right)_{\beta^{\prime} \beta}\right\rangle_{T}\right] \\
& +N_{\mathbf{q}} \sum_{\alpha^{\prime} \beta^{\prime}}\left(\boldsymbol{\Lambda}_{i, \mathbf{q}}^{\dagger}\right)_{\alpha^{\prime} \beta^{\prime}} y \cdot\left[\sum_{\alpha^{\prime \prime}}\left(-\boldsymbol{\Lambda}_{i, \mathbf{q}}\right)_{\alpha \alpha^{\prime \prime}}\left\langle\left(e^{y \hat{\mathbf{C}}_{i}-y \hat{\mathbf{C}}_{i}}\right)_{\alpha^{\prime \prime} \alpha^{\prime} ; \beta^{\prime} \beta}\right\rangle_{T}+\sum_{\beta^{\prime \prime}}\left\langle\left(e^{y \hat{\mathbf{C}}_{i}-y \hat{\mathbf{C}}_{i}}\right)_{\alpha \alpha^{\prime} ; \beta^{\prime} \beta^{\prime \prime}}\right\rangle_{T}\left(\boldsymbol{\Lambda}_{i, \mathbf{q}}\right)_{\beta^{\prime \prime} \beta}\right] .
\end{aligned}
$$

$$
\operatorname{Part} I V:\left\langle\left(\hat{\mathbf{X}}_{i}^{\dagger} \hat{\mathbf{b}}_{\mathbf{q}}^{\dagger} \hat{\mathbf{X}}_{i}^{\dagger}-\hat{\mathbf{b}}_{\mathbf{q}}^{\dagger}\right)_{\alpha \beta}\left(\hat{\mathbf{X}}_{i}^{\dagger} \hat{\mathbf{b}}_{\mathbf{q}} \hat{\mathbf{X}}_{i}^{\dagger}-\hat{\mathbf{b}}_{\mathbf{q}}\right)_{\alpha^{\prime} \beta^{\prime}}\right\rangle_{T}
$$

This last term describes bath-induced interactions between polarons. By using a relation similar to Eq. (A18) and applying it only to the $\hat{\mathbf{X}}_{i}^{\dagger} \hat{\mathbf{b}}_{\mathbf{q}}^{\dagger} \hat{\mathbf{X}}_{i}^{\dagger}$ term, we obtain

$$
\left(\hat{\mathbf{X}}_{i}^{\dagger} \hat{\mathbf{b}}_{\mathbf{q}}^{\dagger} \hat{\mathbf{X}}_{i}^{\dagger}-\hat{\mathbf{b}}_{\mathbf{q}}^{\dagger}\right)=\int_{0}^{1} d x \frac{d}{d x}\left(e^{x \hat{\mathbf{C}}_{i}} \hat{\mathbf{b}}_{\mathbf{q}}^{\dagger} e^{-x \hat{\mathbf{C}}_{i}}\right)=-\int_{0}^{1} d x\left(e^{x \hat{\mathbf{C}}_{i}} \boldsymbol{\Lambda}_{i, \mathbf{q}} e^{-x \hat{\mathbf{C}}_{i}}\right)
$$

and

$$
\begin{aligned}
& \left\langle\left(\hat{\mathbf{X}}_{i}^{\dagger} \hat{\mathbf{b}}_{\mathbf{q}}^{\dagger} \hat{\mathbf{X}}_{i}^{\dagger}-\hat{\mathbf{b}}_{\mathbf{q}}^{\dagger}\right)_{\alpha \beta}\left(\hat{\mathbf{X}}_{i}^{\dagger} \hat{\mathbf{b}}_{\mathbf{q}} \hat{\mathbf{X}}_{i}^{\dagger}-\hat{\mathbf{b}}_{\mathbf{q}}\right)_{\alpha^{\prime} \beta^{\prime}}\right\rangle_{T}=\int_{0}^{1} \int_{0}^{1} d x d y\left\langle\left(e^{x \hat{\mathbf{C}}_{i}} \boldsymbol{\Lambda}_{i, \mathbf{q}} e^{-x \hat{\mathbf{C}}_{i}}\right)_{\alpha \beta}\left(e^{y \hat{\mathbf{C}}_{i}} \boldsymbol{\Lambda}_{i, \mathbf{q}}^{\dagger} e^{-y \hat{\mathbf{C}}_{i}}\right)_{\alpha^{\prime} \beta^{\prime}}\right\rangle_{T} \\
= & \int_{0}^{1} \int_{0}^{1} d x d y \sum_{\gamma \gamma^{\prime}} \sum_{\delta \delta^{\prime}}\left(\boldsymbol{\Lambda}_{i, \mathbf{q}}\right)_{\gamma \gamma^{\prime}}\left(\boldsymbol{\Lambda}_{i, \mathbf{q}}^{\dagger}\right)_{\delta \delta^{\prime}} \cdot\left\langle\left(e^{x \hat{\mathbf{C}}_{i}}\right)_{\alpha \gamma}\left(e^{-x \hat{\mathbf{C}}_{i}}\right)_{\gamma^{\prime} \beta}\left(e^{y \hat{\mathbf{C}}_{i}}\right)_{\alpha^{\prime} \delta}\left(e^{-y \hat{\mathbf{C}}_{i}}\right)_{\delta^{\prime} \beta^{\prime}}\right\rangle_{T}
\end{aligned}
$$

This can thus be calculated by the same method as in Eqs. (A20, A21).

In addition, we would like to point out that the thermal average calculation in Eq. (A9) is exact only when the matrices $\boldsymbol{\Lambda}_{\mathbf{q}}$ commute with each other: $\left[\boldsymbol{\Lambda}_{\mathbf{q}}, \boldsymbol{\Lambda}_{\mathbf{q}^{\prime}}\right]=0$, even though the other derivations are exact for an arbitrary choice of $\Lambda_{\mathbf{q}}$. In order to maintain good control over our two-band polaron transformation method, we can also limit the variational parameters $\boldsymbol{\Lambda}_{\mathbf{q}}$ to be a set of commuting matrices. Though the ground state energy and the contribution from the incoherent part might thus increase, this transformation has the great advantage of an exactly solvable coherent part. Another benefit of using commuting matrices is that the transformed creation/annihilation operators take a simplified form $e^{\hat{S}} \hat{b}_{\mathbf{q}} e^{-\hat{S}}=\hat{b}_{\mathbf{q}}-\sum_{i, \alpha, \beta}\left(\Lambda_{i, \mathbf{q}}^{\alpha \beta}\right)^{*} \hat{a}_{i}^{\alpha \dagger} \hat{a}_{i}^{\beta}$.

\section{Appendix B: Lindblad equation}

After the polaron transformation, the Hamiltonian can be separated into a coherent part Eq. (25) and an incoherent part Eq. (39). This Hamiltonian describes an quantum system (coherent part) coupled to a bosonic bath via interactions (incoherent part). We apply the Lindblad master equation to include the incoherent part.

In the interaction picture, due to $J^{0} ; J^{1} \ll \varepsilon_{\mathrm{P}}^{\Delta}$ for a deep lattice, the polaron operators can be approximated as

$$
\hat{a}_{i}^{\alpha}(t) \approx \hat{a}_{i}^{\alpha} e^{-i \varepsilon_{\mathrm{P}}^{\alpha} t / \hbar} ; \quad \hat{a}_{i}^{\alpha \dagger}(t) \approx \hat{a}_{i}^{\alpha \dagger} e^{+i \varepsilon_{\mathrm{P}}^{\alpha} t / \hbar},
$$


where $\varepsilon_{\mathrm{P}}^{\alpha}$ is the renormalized polaron on-site energy. The interaction (incoherent part) in Eq. (39) is given by:

$$
\begin{aligned}
H_{\mathrm{inc}}(t) & \approx \sum_{\alpha} \sum_{i, j}\left\{B_{i, j}^{\alpha \alpha}(t)\right\} \hat{a}_{i}^{\alpha \dagger} \hat{a}_{j}^{\alpha}+\sum_{\alpha \neq \beta} \sum_{i}\left\{B_{i, i}^{\alpha \beta}(t)\right\} \hat{a}_{i}^{\alpha \dagger} \hat{a}_{i}^{\beta} \cdot e^{-i(\beta-\alpha) \varepsilon_{\mathrm{P}}^{\Delta} t / \hbar} \\
B_{i, j}^{\alpha \alpha}(t) & \equiv-\delta_{j, i \pm 1} J_{\mathrm{P}}^{\alpha} \hat{T}_{i, j}^{\alpha \alpha}(t)+\delta_{i, j} \sum_{\mathbf{q}} \hbar \omega_{\mathbf{q}}\left(\hat{b}_{\mathbf{q}}^{\dagger}(t)+\hat{b}_{-\mathbf{q}}(t)\right)\left(\mathbf{M}_{i, \mathbf{q}}^{\dagger}-\mathbf{\Lambda}_{i, \mathbf{q}}^{\dagger}\right)_{\alpha \alpha} ; \\
B_{i, i}^{\alpha \beta}(t) & \equiv \sum_{\mathbf{q}} \hbar \omega_{\mathbf{q}}\left[\hat{b}_{\mathbf{q}}^{\dagger}(t)\left(\mathbf{M}_{i, \mathbf{q}}^{\dagger}\right)_{\alpha \beta}\left(\hat{K}_{i, i}^{\alpha \beta}(t)\right)+\left(\mathbf{M}_{i, \mathbf{q}}\right)_{\beta \alpha}\left(\hat{K}_{i, i}^{\beta \alpha}(t)\right)^{*} \hat{b}_{\mathbf{q}}(t)\right] \\
& +\sum_{\mathbf{q}} \hbar \omega_{\mathbf{q}}\left[\left(\Lambda_{\mathbf{q}}^{\alpha}\right)\left(\mathbf{M}_{\mathbf{q}}^{\dagger}\right)_{\alpha \beta}\left(\hat{T}_{i, i}^{\alpha \beta}(t)\right)+\left(\Lambda_{\mathbf{q}}^{\beta}\right)^{*}\left(\mathbf{M}_{\mathbf{q}}\right)_{\beta \alpha}\left(\hat{T}_{i, i}^{\beta \alpha}(t)\right)^{*}\right]
\end{aligned}
$$

where $\hat{K}_{i, i}^{\alpha \beta}(t)$ and $\hat{T}_{i, i}^{\alpha \beta}(t)$ are also in the interaction picture. The first term in $H_{\text {inc }}(t)$ describes intra-band dynamics, while the second term describes inter-band dynamics. In both processes, the polaron will emit (absorb) phonons to (from) the bath. Using the general form of the Lindblad equation in Eq. (41), we can define the decoherence factor $\gamma$ as:

$$
\begin{aligned}
\gamma_{i j ; i^{\prime} j^{\prime}}^{\alpha \beta ; \alpha^{\prime} \beta^{\prime}}(\omega) & =\int_{-\infty}^{\infty} d \tau e^{i \omega \tau}\left\langle B_{i j}^{\alpha \beta \dagger}(\tau) B_{i^{\prime} j^{\prime}}^{\alpha^{\prime} \beta^{\prime}}(0)\right\rangle_{T} \\
& =2 \cdot \operatorname{Re}\left(\Gamma_{i j ; i^{\prime} j^{\prime}}^{\alpha \beta ; \alpha^{\prime} \beta^{\prime}}(\omega)\right) \equiv 2 \cdot \operatorname{Re} \int_{0}^{\infty} d \tau e^{i \omega \tau} g_{i j ; i^{\prime} j^{\prime}}^{\alpha \beta ; \beta^{\prime} \beta^{\prime}}(\tau) .
\end{aligned}
$$

By using the rotating wave approximation (RWA), we decouple intra- and inter-band dynamics in the Lindblad equation:

$$
\begin{aligned}
\mathcal{L}_{I}[\rho(t)] & =\sum_{\alpha, \alpha^{\prime}} \sum_{i j} \sum_{i^{\prime} j^{\prime}} \gamma_{i j ; i^{\prime} j^{\prime}}^{\alpha \alpha \alpha^{\prime} \alpha^{\prime}}\left(C_{i^{\prime} j^{\prime}}^{\alpha^{\prime} \alpha^{\prime}} \rho C_{i j}^{\alpha \alpha \dagger}-\frac{1}{2}\left\{C_{i j}^{\alpha \alpha \dagger} C_{i^{\prime} j^{\prime}}^{\alpha^{\prime} \alpha^{\prime}}, \rho\right\}\right) \\
& +\sum_{i} \sum_{i^{\prime}} \gamma_{i i ; i^{\prime} i^{\prime}}^{01 ; 01}\left(C_{i^{\prime} i^{\prime}}^{01} \rho C_{i i}^{01 \dagger}-\frac{1}{2}\left\{C_{i i}^{01 \dagger} C_{i^{\prime} i^{\prime}}^{01}, \rho\right\}\right) \\
& +\sum_{i} \sum_{i^{\prime}} \gamma_{i i ; i^{\prime} i^{\prime}}^{10 ; 10}\left(C_{i^{\prime} i^{\prime}}^{10} \rho C_{i i}^{10 \dagger}-\frac{1}{2}\left\{C_{i i}^{10 \dagger} C_{i^{\prime} i^{\prime}}^{10}, \rho\right\}\right) .
\end{aligned}
$$

The first part $\gamma_{i j ; i^{\prime} j^{\prime}}^{\alpha \alpha ; \alpha^{\prime}}$ describes intra-band decoherence effects. The second part $\gamma_{i i ; i^{\prime} i^{\prime}}^{01 ; 1}$ describes polaron relaxation process from upper to lower band, while the third part $\gamma_{i i ; i^{\prime} i^{\prime}}^{10 ; 10}$ describes an excitation from lower to upper band. We use the short notation $\gamma_{i, j}^{01} \equiv \gamma_{i i ; j j}^{01 ; 01}$ and $C_{i}^{01} \equiv\left(\hat{a}_{i}^{0 \dagger} \hat{a}_{i}^{1}\right)$ for the second term.

In this paper we focus on inter-band relaxation processes. The relaxation rate $\gamma_{i, j}^{01}$ is:

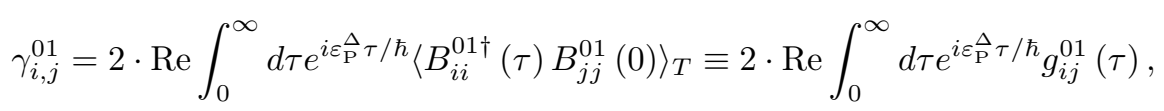

with the correlation function $g_{i j}^{01}(\tau) \equiv g_{i i ; j j}^{01 ; 01}(\tau)$ :

$$
\begin{aligned}
& g_{i j}^{01}(\tau)=\left\langle B_{i i}^{01 \dagger}(\tau) B_{j j}^{01}(0)\right\rangle_{T} \\
&=\sum_{\mathbf{q q}^{\prime}} \hbar^{2} \omega_{\mathbf{q}} \omega_{\mathbf{q}^{\prime}}\left(M_{i, \mathbf{q}}^{01}\right)\left(M_{j, \mathbf{q}^{\prime}}^{01}\right)^{*}\left\langle\left[\left(\hat{K}_{i, i}^{10}(\tau)\right) \hat{b}_{\mathbf{q}}(\tau)+\hat{b}_{-\mathbf{q}}^{\dagger}(\tau)\left(\hat{K}_{i, i}^{10}(\tau)\right)+\left(\Lambda_{i, \mathbf{q}}^{0}-\Lambda_{i, \mathbf{q}}^{1}\right)^{*}\left(\hat{K}_{i, i}^{10}(\tau)-\left\langle\hat{K}_{i, i}^{10}(\tau)\right\rangle_{T}\right)\right]\right. \\
&\left.\cdot\left[\hat{b}_{\mathbf{q}^{\prime}}^{\dagger}(0)\left(\hat{K}_{j, j}^{01}(0)\right)+\left(\hat{K}_{j, j}^{01}(0)\right) \hat{b}_{-\mathbf{q}^{\prime}}(0)+\left(\Lambda_{j, \mathbf{q}^{\prime}}^{0}-\Lambda_{j, \mathbf{q}^{\prime}}^{1}\right)\left(\hat{K}_{j, j}^{01}(0)-\left\langle\hat{K}_{j, j}^{01}(0)\right\rangle_{T}\right)\right]\right\rangle_{T} \cdot \quad(\mathrm{B} 6)
\end{aligned}
$$

From the definition of $\hat{K}_{i, j}^{\alpha \beta}$, we have

$$
\begin{aligned}
\hat{K}_{i, j}^{\alpha \beta}(t) & \equiv \exp \left[\sum_{\mathbf{q}}\left(\mu_{i j, \mathbf{q}}^{\alpha \beta}\right)^{*} \hat{b}_{\mathbf{q}}^{\dagger}(t)-\sum_{\mathbf{q}}\left(\mu_{i j, \mathbf{q}}^{\alpha \beta}\right) \hat{b}_{\mathbf{q}}(t)\right] ; \\
\left(\mu_{i j, \mathbf{q}}^{\alpha \beta}\right) & \equiv-\left(\Lambda_{i, \mathbf{q}}^{\alpha \alpha}\right)+\left(\Lambda_{j, \mathbf{q}}^{\beta \beta}\right),
\end{aligned}
$$


and the thermal averaged results from Bloch identity:

$$
\left\langle\hat{K}_{i, j}^{\alpha \beta}(t)\right\rangle_{T}=\exp \left[-\sum_{\mathbf{q}}\left(N_{\mathbf{q}}+\frac{1}{2}\right)\left(\mu_{i j, \mathbf{q}}^{\alpha \beta}\right)^{*}\left(\mu_{i j, \mathbf{q}}^{\alpha \beta}\right)\right],
$$

as well as:

$$
\begin{array}{r}
\left\langle\hat{K}_{i, j}^{\alpha \beta}(t) \hat{K}_{i^{\prime}, j^{\prime}}^{\alpha^{\prime} \beta^{\prime}}(0)\right\rangle_{T}=\exp \left[-\sum_{\mathbf{q}}\left(N_{\mathbf{q}}+\frac{1}{2}\right)\left(\mu_{i j, \mathbf{q}}^{\alpha \beta}\right)^{*}\left(\mu_{i j, \mathbf{q}}^{\alpha \beta}\right)\right] \exp \left[-\sum_{\mathbf{q}}\left(N_{\mathbf{q}}+\frac{1}{2}\right)\left(\mu_{i^{\prime} j^{\prime}, \mathbf{q}}^{\alpha^{\prime} \beta^{\prime}}\right)^{*}\left(\mu_{i^{\prime} j^{\prime}, \mathbf{q}}^{\alpha^{\prime} \beta^{\prime}}\right)\right] \\
\cdot \exp \left[-\sum_{\mathbf{q}}\left(N_{\mathbf{q}}\right)\left(\mu_{i j, \mathbf{q}}^{\alpha \beta}\right)^{*}\left(\mu_{i^{\prime} j^{\prime}, \mathbf{q}}^{\alpha^{\prime} \beta^{\prime}}\right) e^{i \omega_{\mathbf{q}} t}-\sum_{\mathbf{q}}\left(N_{\mathbf{q}}+1\right)\left(\mu_{i j, \mathbf{q}}^{\alpha \beta}\right)\left(\mu_{i^{\prime} j^{\prime}, \mathbf{q}}^{\alpha^{\prime} \beta^{\prime}}\right)^{*} e^{-i \omega_{\mathbf{q}} t}\right] .
\end{array}
$$

The elements such as $\left\langle\hat{b}_{\mathbf{q}}^{\dagger}(t) \hat{K}_{i, j}^{\alpha \beta}(t)\right\rangle_{T}$ can be obtained by using the Glauber formula $e^{\hat{A}+\hat{B}}=e^{\hat{A}} e^{\hat{B}} e^{-\frac{1}{2}[\hat{A}, \hat{B}]}($ with the condition $[\hat{A},[\hat{A}, \hat{B}]]=[\hat{B},[\hat{A}, \hat{B}]]=0)$ and differentiating $\hat{K}_{i, j}^{\alpha \beta}(t)$ in Eq. (B7) as:

$$
\begin{aligned}
& \frac{\partial}{\partial\left(\mu_{i j, \mathbf{q}}^{\alpha \beta}\right)^{*}} \hat{K}_{i, j}^{\alpha \beta}(t)=\left[\hat{b}_{\mathbf{q}}^{\dagger}(t)-\frac{1}{2}\left(\mu_{i j, \mathbf{q}}^{\alpha \beta}\right)\right] \hat{K}_{i, j}^{\alpha \beta}(t) \\
& \frac{\partial}{\partial\left(\mu_{i j, \mathbf{q}}^{\alpha \beta}\right)} \hat{K}_{i, j}^{\alpha \beta}(t)=\hat{K}_{i, j}^{\alpha \beta}(t)\left[-\hat{b}_{\mathbf{q}}(t)-\frac{1}{2}\left(\mu_{i j, \mathbf{q}}^{\alpha \beta}\right)^{*}\right] .
\end{aligned}
$$

As an example, averages of $\left\langle\hat{b}_{\mathbf{q}}^{\dagger}(t) \hat{K}_{i, j}^{\alpha \beta}(t)\right\rangle_{T}$ in Eq. (B6) can then be obtained by using Eq. (B10) and switching the order of differentiation and thermal averaging:

$$
\begin{aligned}
\left\langle\hat{b}_{\mathbf{q}}^{\dagger}(t) \hat{K}_{i, j}^{\alpha \beta}(t)\right\rangle_{T} & =\frac{\partial}{\partial\left(\mu_{i j, \mathbf{q}}^{\alpha \beta}\right)^{*}}\left\langle\hat{K}_{i, j}^{\alpha \beta}(t)\right\rangle_{T}+\frac{1}{2}\left(\mu_{i j, \mathbf{q}}^{\alpha \beta}\right)\left\langle\hat{K}_{i, j}^{\alpha \beta}(t)\right\rangle_{T} \\
& =-N_{\mathbf{q}}\left(\mu_{i j, \mathbf{q}}^{\alpha \beta}\right) \exp \left[-\sum_{\mathbf{q}}\left(N_{\mathbf{q}}+\frac{1}{2}\right)\left(\mu_{i j, \mathbf{q}}^{\alpha \beta}\right)^{*}\left(\mu_{i j, \mathbf{q}}^{\alpha \beta}\right)\right] .
\end{aligned}
$$

Finally the correlation function Eq. (B6) for inter-band relaxation is:

$$
\begin{aligned}
g_{i j}^{01}(\tau)= & \sum_{\mathbf{q}}\left(\hbar \omega_{\mathbf{q}}\left|M_{\mathbf{q}}^{01}\right|\right)^{2} \cos \left[q_{x}(i-j) d\right]\left[\left(N_{\mathbf{q}}+1\right) e^{-i \omega_{\mathbf{q}} \tau}+\left(N_{\mathbf{q}}\right) e^{+i \omega_{\mathbf{q}} \tau}\right]\left\langle\hat{K}_{i, i}^{10}(\tau) \hat{K}_{j, j}^{01}(0)\right\rangle_{T} \\
& +\sum_{\mathbf{q} \mathbf{q}^{\prime}} \hbar^{2} \omega_{\mathbf{q}} \omega_{\mathbf{q}^{\prime}}\left(M_{\mathbf{q}}^{01}\right)\left(M_{\mathbf{q}^{\prime}}^{01}\right)\left(\Lambda_{\mathbf{q}}^{0}-\Lambda_{\mathbf{q}}^{1}\right)\left(\Lambda_{\mathbf{q}^{\prime}}^{0}-\Lambda_{\mathbf{q}^{\prime}}^{1}\right) \sin \left[q_{x}(i-j) d\right] \sin \left[q_{x}^{\prime}(i-j) d\right] \\
& \cdot\left[\left(N_{\mathbf{q}}+1\right) e^{-i \omega_{\mathbf{q}} \tau}-\left(N_{\mathbf{q}}\right) e^{+i \omega_{\mathbf{q}} \tau}\right] \cdot\left[\left(N_{\mathbf{q}^{\prime}}+1\right) e^{-i \omega_{\mathbf{q}^{\prime}} \tau}-\left(N_{\mathbf{q}^{\prime}}\right) e^{+i \omega_{\mathbf{q}^{\prime}} \tau}\right]\left\langle\hat{K}_{i, i}^{10}(\tau) \hat{K}_{j, j}^{01}(0)\right\rangle_{T}
\end{aligned}
$$

with

$$
\begin{aligned}
& \left\langle\hat{K}_{i, i}^{10}(\tau) \hat{K}_{j, j}^{01}(0)\right\rangle_{T} \\
= & \exp \left\{\sum_{\mathbf{q}}\left(\Lambda_{\mathbf{q}}^{0}-\Lambda_{\mathbf{q}}^{1}\right)^{2}\left[-\left(2 N_{\mathbf{q}}+1\right)+\left(N_{\mathbf{q}}\right) \cos \left[q_{x}(i-j) d\right] e^{+i \omega_{\mathbf{q}} \tau}+\left(N_{\mathbf{q}}+1\right) \cos \left[q_{x}(i-j) d\right] e^{-i \omega_{\mathbf{q}} \tau}\right]\right\} .
\end{aligned}
$$

The first term in correlation function (B12) is a single-phonon process, which is similar to Fermi's Golden Rule except a renormalization factor $\left\langle\hat{K}_{i, i}^{10}(\tau) \hat{K}_{j, j}^{01}(0)\right\rangle_{T}$. The second term describes higher order process involving two phonons, which are absent in Fermi's Golden Rule.

The corresponding relaxation rate $\gamma_{i, j}^{01}$ is calculated by Eq. (B5). At zero temperature, this corresponds to spontaneous relaxation process:

$$
\begin{aligned}
\gamma_{i, j}^{01}=2 & \cdot \operatorname{Re} \int_{0}^{\infty} d \tau e^{i \varepsilon_{\mathbf{P}}^{\Delta} \tau / \hbar} \cdot \sum_{\mathbf{q}}\left(\hbar \omega_{\mathbf{q}}\left|M_{\mathbf{q}}^{01}\right|\right)^{2} \cos \left[q_{x}(i-j) d\right] e^{-i \omega_{\mathbf{q}} \tau} \cdot\left\langle\hat{K}_{i, i}^{10}(\tau) \hat{K}_{j, j}^{01}(0)\right\rangle_{T} \\
+ & 2 \cdot \operatorname{Re} \int_{0}^{\infty} d \tau e^{i \varepsilon_{\mathbf{P}}^{\Delta} \tau / \hbar} \cdot \sum_{\mathbf{q} \mathbf{q}^{\prime}} \hbar^{2} \omega_{\mathbf{q}} \omega_{\mathbf{q}^{\prime}}\left(M_{\mathbf{q}}^{01}\right)\left(M_{\mathbf{q}^{\prime}}^{01}\right)\left(\Lambda_{\mathbf{q}}^{0}-\Lambda_{\mathbf{q}}^{1}\right)\left(\Lambda_{\mathbf{q}^{\prime}}^{0}-\Lambda_{\mathbf{q}^{\prime}}^{1}\right) \\
& \cdot \sin \left[q_{x}(i-j) d\right] \sin \left[q_{x}^{\prime}(i-j) d\right] e^{-i \omega_{\mathbf{q}} \tau} e^{-i \omega_{\mathbf{q}^{\prime}} \tau} \cdot\left\langle\hat{K}_{i, i}^{10}(\tau) \hat{K}_{j, j}^{01}(0)\right\rangle_{T} .
\end{aligned}
$$


The second part, which involves higher order correlations with two phonons being emitted, is neglected in the total decay rate in Eq. (44).

[1] R. P. Feynman, Int. J. Theor. Phys. 21, 467 (1982).

[2] S. Lloyd, Science 273, 1073 (1996).

[3] D. Jaksch, C. Bruder, J. Cirac, C. Gardiner, and P. Zoller, Phys. Rev. Lett. 81, 3108 (1998).

[4] J. I. Cirac and P. Zoller, Nat. Phys. 8, 264 (2012).

[5] I. Bloch, J. Dalibard, and W. Zwerger, Rev. Mod. Phys. 80, 885 (2008).

[6] I. Bloch, J. Dalibard, and S. Nascimbène, Nat. Phys. 8, 267 (2012).

[7] T. Gericke, P. Würtz, D. Reitz, T. Langen, and H. Ott, Nat. Phys. 4, 949 (2008).

[8] W. S. Bakr, J. I. Gillen, A. Peng, S. Fölling, and M. Greiner, Nature 462, 74 (2009).

[9] J. F. Sherson, C. Weitenberg, M. Endres, M. Cheneau, I. Bloch, and S. Kuhr, Nature 467, 68 (2010).

[10] F. Serwane, G. Zürn, T. Lompe, T. B. Ottenstein, a. N. Wenz, and S. Jochim, Science 332, 336 (2011).

[11] Y.-J. Lin, R. L. Compton, K. Jiménez-García, J. V. Porto, and I. B. Spielman, Nature 462, 628 (2009).

[12] D. Cocks, P. P. Orth, S. Rachel, M. Buchhold, K. Le Hur, and W. Hofstetter, Phys. Rev. Lett. 109, 205303 (2012).

[13] F. Herrera and R. V. Krems, Phys. Rev. A 84, 051401 (2011).

[14] S. Mostame, P. Rebentrost, A. Eisfeld, A. J. Kerman, D. I. Tsomokos, and A. Aspuru-Guzik, New J. Phys. 14, 105013 (2012).

[15] J. P. Hague and C. MacCormick, Phys. Rev. Lett. 109, 223001 (2012).

[16] J. P. Hague and C. MacCormick, New J. Phys. 14, 033019 (2012).

[17] L. Mathey, D.-W. Wang, W. Hofstetter, M. Lukin, and E. Demler, Phys. Rev. Lett. 93, 120404 (2004).

[18] A. N. Das and S. Sil, J. Phys. Condens. Matter 5, 8265 (1999).

[19] D. J. J. Marchand, G. De Filippis, V. Cataudella, M. Berciu, N. Nagaosa, N. V. Prokof'ev, A. S. Mishchenko, and P. C. E. Stamp, Phys. Rev. Lett. 105, 266605 (2010).

[20] T. Holstein, Ann. Phys. (N. Y). 8, 343 (1959).

[21] G. D. Mahan, Many-Particle Physics (Springer US, 2000).

[22] M. Hohenadler, H. G. Evertz, and W. von der Linden, Phys. Rev. B 69, 024301 (2004).

[23] D. Golež, J. Bonča, L. Vidmar, and S. A. Trugman, Phys. Rev. Lett. 109, 236402 (2012).

[24] D. J. J. Marchand and M. Berciu, Phys. Rev. B 88, 060301 (2013).

[25] W. Zwerger, Z. Phys. B - Condens. Matter 53, 53 (1983).

[26] A. Leggett, S. Chakravarty, A. T. Dorsey, M. P. A. Fisher, A. Garg, and W. Zwerger, Rev. Mod. Phys. 59, 1 (1987).

[27] K. Agarwal, I. Martin, M. Lukin, and E. Demler, Phys. Rev. B 87, 144201 (2013).

[28] S. Bera, A. Nazir, A. W. Chin, H. U. Baranger, and S. Florens, Phys. Rev. B 90, 075110 (2014).
[29] J. Zaanen and A. M. Oles, Phys. Rev. B 37, 9423 (1988).

[30] J. T. Gammel, R. J. Donohoe, A. R. Bishop, and B. I. Swanson, Phys. Rev. B 42, 10566 (1990).

[31] Y. Furukawa and M. Tasumi, Int. Conf. Sci. Technol. Synth. Met. 69, 629 (1994).

[32] A. Lanzara, P. V. Bogdanov, X. J. Zhou, S. A. Kellar, D. L. Feng, E. D. Lu, T. Yoshida, H. Eisaki, A. Fujimori, K. Kishio, J. I. Shimoyama, T. Noda, S. Uchida, Z. Hussain, and Z. X. Shen, Nature 412, 510 (2001).

[33] J. Devreese and S. Alexandrov, Reports Prog. Phys. 72, 066501 (2009).

[34] A. S. Alexandrov and J. T. Devreese, Advances in Polaron Physics, Springer Series in Solid-State Sciences, Vol. 159 (2010).

[35] M. Y. Kagan and V. V. Val'kov, J. Supercond. Nov. Magn. 25, 1379 (2012).

[36] V. M. Stojanović, T. Shi, C. Bruder, and J. I. Cirac, Phys. Rev. Lett. 109, 250501 (2012).

[37] A. Grier, M. Cetina, F. Oručević, and V. Vuletić, Phys. Rev. Lett. 102, 223201 (2009).

[38] C. Zipkes, S. Palzer, C. Sias, and M. Köhl, Nature 464, 388 (2010).

[39] S. Schmid, A. Härter, and J. H. Denschlag, Phys. Rev. Lett. 105, 133202 (2010).

[40] W. G. Rellergert, S. T. Sullivan, S. Kotochigova, A. Petrov, K. Chen, S. J. Schowalter, and E. R. Hudson, Phys. Rev. Lett. 107, 243201 (2011).

[41] L. Ratschbacher, C. Zipkes, C. Sias, and M. Köhl, Nat. Phys. 8, 649 (2012).

[42] L. Ratschbacher, C. Sias, L. Carcagni, J. Silver, C. Zipkes, and M. Köhl, Phys. Rev. Lett. 110, 160402 (2013).

[43] J. Casanova, A. Mezzacapo, L. Lamata, and E. Solano, Phys. Rev. Lett. 108, 190502 (2012).

[44] K. Günter, T. Stöferle, H. Moritz, M. Köhl, and T. Esslinger, Phys. Rev. Lett. 96, 180402 (2006).

[45] S. Ospelkaus, C. Ospelkaus, O. Wille, M. Succo, P. Ernst, K. Sengstock, and K. Bongs, Phys. Rev. Lett. 96, 180403 (2006).

[46] T. Best, S. Will, U. Schneider, L. Hackermüller, D. van Oosten, I. Bloch, and D.-S. Lühmann, Phys. Rev. Lett. 102, 030408 (2009).

[47] S. Will, T. Best, S. Braun, U. Schneider, and I. Bloch, Phys. Rev. Lett. 106, 115305 (2011).

[48] M. J. Leskinen, O. H. T. Nummi, F. Massel, and P. Törmä, New J. Phys. 12, 073044 (2010).

[49] W. Casteels, J. Tempere, and J. T. Devreese, Phys. Rev. A 86, 043614 (2012).

[50] N. Prokof'ev and B. Svistunov, Phys. Rev. B 77, 125101 (2008).

[51] C. Mora and F. Chevy, Phys. Rev. A 80, 033607 (2009).

[52] A. Schirotzek, C.-H. Wu, A. Sommer, and M. W. Zwierlein, Phys. Rev. Lett. 102, 230402 (2009).

[53] S. Nascimbène, N. Navon, K. J. Jiang, L. Tarruell, M. Teichmann, J. McKeever, F. Chevy, and C. Salomon, Phys. Rev. Lett. 103, 170402 (2009).

[54] W. Yi and W. Zhang, Phys. Rev. Lett. 109, 140402 (2012). 
[55] P. Massignan, M. Zaccanti, and G. M. Bruun, Reports Prog. Phys. 77, 034401 (2014).

[56] M. Cetina, M. Jag, R. S. Lous, J. T. M. Walraven, R. Grimm, R. S. Christensen, and G. M. Bruun, Phys. Rev. Lett. 115, 135302 (2015).

[57] Z. Lan and C. Lobo, Phys. Rev. A 92, 053605 (2015).

[58] A. Klein and M. Fleischhauer, Phys. Rev. A 71, 033605 (2005).

[59] F. M. Cucchietti and E. Timmermans, Phys. Rev. Lett. 96, 210401 (2006).

[60] M. Bruderer, A. Klein, S. Clark, and D. Jaksch, Phys. Rev. A 76, 011605 (2007).

[61] M. Bruderer, A. Klein, S. R. Clark, and D. Jaksch, New J. Phys. 10, 033015 (2008).

[62] J. Tempere, W. Casteels, M. Oberthaler, S. Knoop, E. Timmermans, and J. Devreese, Phys. Rev. B 80, 184504 (2009).

[63] B. Gadway, D. Pertot, R. Reimann, and D. Schneble, Phys. Rev. Lett. 105, 045303 (2010).

[64] A. Privitera and W. Hofstetter, Phys. Rev. A 82, 063614 (2010).

[65] T. Fukuhara, A. Kantian, M. Endres, M. Cheneau, P. Schauß, S. Hild, D. Bellem, U. Schollwöck, T. Giamarchi, C. Gross, I. Bloch, and S. Kuhr, Nat. Phys. 9, 235 (2013).

[66] W. Casteels, J. Tempere, and J. T. Devreese, Phys. Rev. A 88, 013613 (2013).

[67] S. Dutta and E. J. Mueller, Phys. Rev. A 88, 053601 (2013).

[68] S. P. Rath and R. Schmidt, Phys. Rev. A 88, 053632 (2013).

[69] D. Benjamin and E. Demler, Phys. Rev. A 89, 033615 (2014).

[70] E. Compagno, G. D. Chiara, D. G. Angelakis, and G. M. Palma, arXiv:1410.8833 (2014).

[71] W. Li and S. Das Sarma, Phys. Rev. A 90, 013618 (2014).

[72] A. Shashi, F. Grusdt, D. A. Abanin, and E. Demler, Phys. Rev. A 89, 053617 (2014).

[73] F. Grusdt, A. Shashi, D. Abanin, and E. Demler, Phys. Rev. A 90, 063610 (2014).

[74] F. Grusdt, Y. E. Shchadilova, A. N. Rubtsov, and E. Demler, Sci. Rep. 5, 12124 (2015).

[75] Y. E. Shchadilova, F. Grusdt, A. N. Rubtsov, and E. Demler, arXiv:1410.5691 (2014).

[76] R. S. Christensen, J. Levinsen, and G. M. Bruun, Phys. Rev. Lett. 115, 160401 (2015).

[77] J. Levinsen, M. M. Parish, and G. M. Bruun, Phys. Rev. Lett. 115, 125302 (2015).

[78] R. Schmidt and M. Lemeshko, Phys. Rev. Lett. 114, 203001 (2015).

[79] M. Hohmann, F. Kindermann, B. Gänger, T. Lausch, D. Mayer, F. Schmidt, and A. Widera, EPJ Quantum Technol. 2, 23 (2015).

[80] L. A. P. Ardila and S. Giorgini, Phys. Rev. A 92, 33612 (2015).

[81] F. Grusdt and E. Demler, arXiv:1510.04934 (2015).

[82] G. Pupillo, A. Griessner, A. Micheli, M. Ortner, D.W. Wang, and P. Zoller, Phys. Rev. Lett. 100, 050402 (2008).

[83] M. Ortner, A. Micheli, G. Pupillo, and P. Zoller, New J. Phys. 11, 055045 (2009).

[84] F. Herrera, K. W. Madison, R. V. Krems, and M. Berciu, Phys. Rev. Lett. 110, 223002 (2013).
[85] M. Gullans, T. G. Tiecke, D. E. Chang, J. Feist, J. D. Thompson, J. I. Cirac, P. Zoller, and M. D. Lukin, Phys. Rev. Lett. 109, 235309 (2012).

[86] R. Mottl, F. Brennecke, K. Baumann, R. Landig, T. Donner, and T. Esslinger, Science 336, 1570 (2012).

[87] U. Bissbort, D. Cocks, A. Negretti, Z. Idziaszek, T. Calarco, F. Schmidt-Kaler, W. Hofstetter, and R. Gerritsma, Phys. Rev. Lett. 111, 080501 (2013).

[88] J. Heinze, S. Götze, J. S. Krauser, B. Hundt, N. Fläschner, D. S. Lühmann, C. Becker, and K. Sengstock, Phys. Rev. Lett. 107, 135303 (2011).

[89] N. Spethmann, F. Kindermann, S. John, C. Weber, D. Meschede, and A. Widera, Phys. Rev. Lett. 109, 235301 (2012).

[90] R. Scelle, T. Rentrop, A. Trautmann, T. Schuster, and M. K. Oberthaler, Phys. Rev. Lett. 111, 070401 (2013).

[91] D. Chen, C. Meldgin, and B. DeMarco, Phys. Rev. A 90, 013602 (2014).

[92] E. Braaten and H. W. Hammer, Phys. Rep. 428, 259 (2006).

[93] M. Olshanii, Phys. Rev. Lett. 81, 938 (1998).

[94] P. Massignan and Y. Castin, Phys. Rev. A 74, 013616 (2006).

[95] Y. Nishida and S. Tan, Phys. Rev. Lett. 101, 170401 (2008).

[96] A. Daley, P. Fedichev, and P. Zoller, Phys. Rev. A 69, 022306 (2004).

[97] R. A. Harris and R. Silbey, J. Chem. Phys. 83, 1069 (1985).

[98] J. Chatterjee and A. N. Das, Phys. Rev. B 61, 4592 (2000).

[99] V. M. Stojanović, P. A. Bobbert, and M. A. J. Michels, Phys. Rev. B 69, 144302 (2004).

[100] P. Barone, R. Raimondi, M. Capone, and C. Castellani, Phys. Rev. B 73, 085120 (2006).

[101] R. Silbey and R. W. Munn, J. Chem. Phys. 72, 2763 (1980).

[102] R. W. Munn and R. Silbey, J. Chem. Phys. 83, 1843 (1985).

[103] R. W. Munn and R. Silbey, J. Chem. Phys. 83, 1854 (1985).

[104] D. Chen, J. Ye, H. Zhang, and Y. Zhao, J. Phys. Chem. B 115, 5312 (2011).

[105] C. A. Parra-Murillo, J. Madroñero, and S. Wimberger, Phys. Rev. A 88, 032119 (2013).

[106] C. A. Parra-Murillo, J. Madroñero, and S. Wimberger, Phys. Rev. A 89, 053610 (2014).

[107] D. H. Santamore and E. Timmermans, New J. Phys. 13, 103029 (2011).

[108] T. Yin, P. Zhang, and W. Zhang, Phys. Rev. A 84, 052727 (2011).

[109] E. Malatsetxebarria, Z. Cai, U. Schollwöck, and M. Cazalilla, Phys. Rev. A 88, 063630 (2013).

[110] Z. Lan and C. Lobo, Phys. Rev. A 90, 033627 (2014).

[111] Z. Cai, U. Schollwöck, and L. Pollet, Phys. Rev. Lett. 113, 260403 (2014).

[112] A. Griessner, A. Daley, S. Clark, D. Jaksch, and P. Zoller, Phys. Rev. Lett. 97, 220403 (2006).

[113] A. Griessner, A. J. Daley, S. R. Clark, D. Jaksch, and P. Zoller, New J. Phys. 9, 44 (2007).

[114] D. K. K. Lee and J. M. F. Gunn, Phys. Rev. B 46, 301 (1992).

[115] R. Kalas and D. Blume, Phys. Rev. A 73, 043608 (2006).

[116] D.-S. Lühmann, K. Bongs, K. Sengstock, and 
D. Pfannkuche, Phys. Rev. Lett. 101, 050402 (2008).

[117] M. Bruderer, W. Bao, and D. Jaksch, EPL 82, 7 (2008).

[118] D. Roberts and S. Rica, Phys. Rev. Lett. 102, 025301 (2009).
[119] G. Grosso and G. P. Parravicini, Solid State Physics, 2nd Edition (2013) p. 434. 